$\mathrm{MZ}-\mathrm{TH} / 05-04$

hep-ph/0502194

\title{
Collinear Subtractions in Hadroproduction of Heavy Quarks
}

\author{
B. A. Kniehl, G. Kramer, I. Schienbein \\ II. Institut für Theoretische Physik, Universität Hamburg, \\ Luruper Chaussee 149, D-22761 Hamburg, Germany \\ E-mail: kniehl@mail.desy.de, kramer@mail.desy.de, schien@mail.desy.de \\ and \\ H. Spiesberger \\ Institut für Physik, Johannes-Gutenberg-Universität, \\ Staudinger Weg 7, D-55099 Mainz, Germany \\ E-mail: hspiesb@thep.physik.uni-mainz.de
}

\begin{abstract}
We present a detailed discussion of the collinear subtraction terms needed to establish a massive variable-flavour-number scheme for the one-particle inclusive production of heavy quarks in hadronic collisions. The subtraction terms are computed by convoluting appropriate partonic cross sections with perturbative parton distribution and fragmentation functions relying on the method of mass factorization. We find (with one minor exception) complete agreement with the subtraction terms obtained in a previous publication by comparing the zero-mass limit of a fixed-order calculation with the genuine massles results in the $\overline{\mathrm{MS}}$ scheme. This presentation will be useful for extending the massive variable-flavour-number scheme to other processes.
\end{abstract}




\section{Introduction}

Heavy-quark production in highly energetic $e^{+} e^{-}, \gamma \gamma, \gamma p, e p$ and $p \bar{p}$ collisions has attracted much interest in the past few years, both experimentally and theoretically. Heavy quarks are those with masses $m \gg \Lambda_{\mathrm{QCD}}$ so that $\alpha_{s}(m) \ll 1$, where $\alpha_{s}\left(\mu_{R}\right)$ is the strongcoupling constant at renormalization scale $\mu_{R}$. According to this definition, the charm, bottom and top quarks $(c, b, t)$ are heavy whereas the up, down and strange quarks $(u, d, s)$ are light. Since $\alpha_{s}(m) \ll 1$, heavy-quark production is a calculable process in perturbative QCD. The heavy-quark mass acts as a cutoff for initial- and final-state collinear singularities and sets the scale for the perturbative expansion in $\alpha_{s}$.

On this basis, most of the next-to-leading-order (NLO) QCD calculations have been performed in the past [1, 2, 3, 4, Corresponding results are reliable when $m$ is the only large scale, as for example in calculations of the total cross section or if any additional scale, for example the transverse momentum $p_{T}$ of the produced heavy quark in $\gamma \gamma, \gamma p$ and $p \bar{p}$ reactions or the lepton momentum transfer $Q$ in deep-inelastic ep scattering (DIS), is not much larger than the mass $m$. However, when $p_{T}$ (or $Q$ ) is much larger than the mass, large logarithms $\ln \left(p_{T}^{2} / m^{2}\right)$ or $\ln \left(Q^{2} / m^{2}\right)$ arise to all orders, so that fixed-order perturbation theory is no longer valid. As is well known, these logarithms can be resummed and, this way, the perturbation series can be improved.

The isolation and resummation of large logarithms is similar to the conventional massless parton model approach, where initial- or final-state collinear singularities are absorbed into parton distribution functions (PDF) of the incoming hadrons or photons and into fragmentation functions (FF) for the produced light hadrons (or photons), respectively. Therefore, this approach is usually referred to as zero-mass variable-flavour-number scheme (ZM-VFNS). The notion "variable flavour number" is used since, in the parton model, the number of active quark flavours is increased by one unit, $n_{f} \rightarrow n_{f}+1$, when the factorization scale crosses certain transition scales (which are usually taken to be of the order of the heavy-quark mass) ${ }^{1}$. In contrast, the fixed-order treatment, where $m$ is kept as a large scale, is called the fixed-flavour-number scheme (FFNS), since the number of flavours in the initial state is fixed to $n_{f}=3$ (4) for charm (bottom) production. One can combine cross sections calculated in the FFNS after certain modifications with heavyquark FFs and PDFs which contain the resummed large logarithms. This prescription is called the massive or general-mass VFNS (GM-VFNS) ${ }^{2}$.

One might expect that the partonic cross sections calculated in the FFNS approach the corresponding ZM-VFNS cross sections in the limit $m \rightarrow 0$ if the collinear singular terms proportional to $\ln \left(\mathrm{m}^{2} / \mathrm{s}\right)$ are subtracted, i.e., the subtracted FFNS cross sections differ from the ZM-VFNS cross sections only by terms $\sim m^{2} / p_{T}^{2}$. If this was true, the FFNS and ZM-VFNS results for the cross sections would approach each other for $p_{T}^{2} \gg m^{2}$. This expectation, however, is not true, as was first demonstrated by Mele and Nason

\footnotetext{
${ }^{1}$ For a detailed discussion see the appendix in Ref. [5] and references given there.

${ }^{2}$ For details see, e.g., Refs. [6, 7].
} 
8] for inclusive heavy-quark production in $e^{+} e^{-}$annihilation at $\mathrm{NLO}\left(e^{+} e^{-} \rightarrow Q \bar{Q} g\right.$, where $Q$ is the heavy quark). They found that the limit $m \rightarrow 0$ of the cross section for $e^{+} e^{-} \rightarrow Q \bar{Q} g$ and the cross section calculated with $m=0$ from the beginning (in the $\overline{\mathrm{MS}}$ scheme) differ by finite terms of $\mathcal{O}\left(\alpha_{s}\right)$. The reason for the occurrence of these finite terms is the different definition of the collinear singular terms in the two approaches. In the ZM-VFNS calculation, the heavy-quark mass is set to zero from the beginning and the collinearly divergent terms are defined with the help of dimensional regularization. This fixes the finite terms in a specific way (in a given factorization scheme), and their form is inherent to the chosen regularization procedure. If, on the other hand, one starts with $m \neq 0$ and performs the limit $m \rightarrow 0$ afterwards, the finite terms can be different. In Ref. 8], it was shown that these additional finite terms emerging in the limit $m \rightarrow 0$ out of the theory with $m \neq 0$ can be generated in the theory for $m=0$ with $\overline{\mathrm{MS}}$ factorization by convoluting this cross section with a partonic fragmentation function $d_{Q \rightarrow Q}(x, \mu)$ for the transition from massless to massive heavy quarks $Q$ (the explicit form of $d_{Q \rightarrow Q}(x, \mu)$ will be given later).

If this interpretation of the finite terms in the theory with $m \neq 0$ as partonic $\mathrm{FF}$ is generally true, then $d_{Q \rightarrow Q}(x, \mu)$ should be process independent and could be used in any other heavy-quark production process. The universality of the partonic $\mathrm{FF}$ has been confirmed by performing the same calculation as in Ref. [8] for the process $\gamma^{\star} Q \rightarrow Q g$ [9, 10, where $\gamma^{\star}$ is a space-like virtual photon, $\gamma \gamma \rightarrow Q \bar{Q} g$ [11] and $g g \rightarrow Q \bar{Q} g$ [12] and showing that the finite terms are obtained from a convolution of the corresponding LO cross sections with $d_{Q \rightarrow Q}(x, \mu)$. The process-independence of $d_{Q \rightarrow Q}(x, \mu)$ was established on more general grounds in Ref. 13. Moreover, process-independent derivations of the partonic FFs have been performed by Ma [14] and recently by Melnikov and Mitov [15, 16], who have computed the partonic FFs to $\mathcal{O}\left(\alpha_{s}^{2}\right)$.

The fact that the theory with $m \neq 0$ and the ZM-VFNS are related by the convolution of the ZM-VFNS cross section with partonic FFs has been used in several ways. In Ref. 8], $d_{Q \rightarrow Q}\left(x, \mu_{0}\right)$ was used as the initial condition, at $\mu=\mu_{0}=\mathcal{O}(m)$, for the calculation of $d_{Q \rightarrow Q}(x, \mu)$ at an arbitrary scale $\mu$ with the standard evolution equation. Later, Cacciari and Greco calculated with the same procedure the cross section for heavyquark production in $p \bar{p}$ and $p p$ collisions from the ZM-VFNS cross section supplemented with evolved $d_{Q \rightarrow Q}(x, \mu)$ as a function of $p_{T}$ [17. Partonic FFs used together with a zeromass hard-scattering cross section have subsequently been applied also to heavy-quark production in $\gamma \gamma$ [18] and $\gamma p$ [19, 20] processes. In Ref. [20, the approach was generalized to the reaction $\gamma+p \rightarrow D^{\star}+X$. The transition $c \rightarrow D^{\star}$ was described by a FF containing besides a non-perturbative contribution the purely perturbative partonic FF. The nonperturbative part was described by a function containing two parameters which were fixed by comparison to experimental data for $e^{+}+e^{-} \rightarrow D^{\star}+X$. In Refs. [17, 18, 19, 20], the perturbative $\mathrm{FF}$ approach was motivated by the requirement to match the ZM-VFNS as close as possible to the $m \neq 0$ theory. This could be achieved since at small $p_{T}=\mathcal{O}(m)$ the evolution of $d_{Q \rightarrow Q}\left(x, \mu_{0}\right)$ was not yet effective and, therefore, $d_{Q \rightarrow Q}$ was just taking account of the difference of the two theories. However, terms proportional to $m^{2} / p_{T}^{2}$ are 
not included in this way.

The so-called FONLL approach [21, 22, 23, 24, 25] is an attempt to repair this deficiency. There, the ZM-VFNS with perturbative FFs together with a non-perturbative component was combined with the FFNS with $n_{f}=3$ (4) for charm (bottom) production, introducing a $p_{T}$ dependent suppression factor by hand. In addition, $m^{2} / p_{T}^{2}$ terms have been included in extensions of the ACOT scheme [26, 27] to one-particle inclusive production of $D$ mesons in charged-current and neutral-current DIS [10, 28. In Ref. [29], the ACOT scheme has been applied to one-particle inclusive heavy-quark production in $p \bar{p}$ collisions.

Instead of incorporating the finite terms $d_{Q \rightarrow Q}(x, \mu)$ into the initial conditions of the perturbative FFs at $\mu=\mu_{0}=\mathcal{O}(m)$, one can take this difference also into account by switching to a new factorization scheme, which we call the massive factorization scheme. In this scheme, starting from the ZM-VFNS, one adjusts the factorization of the finalstate collinear singularities associated with the massive quarks in such a way that it matches the massive calculation in the limit $m \rightarrow 0$. Of course, the hard-scattering cross sections of any other process for inclusive $D^{\star}$ production must be transformed to the new scheme as well. This is particularly important for the reaction $e^{+}+e^{-} \rightarrow$ $D^{\star}+X$ from which the information on the non-perturbative FF for $c \rightarrow D^{\star}$ is obtained by comparison to experimental data. So far, calculations in this massive factorization scheme were performed for $\gamma+p \rightarrow D^{\star}+X$ in Ref. [30], where also fits of the new FFs for $c \rightarrow D^{\star}$ have been presented (for a comparison of FFs in the massive and the $\overline{\mathrm{MS}}$ scheme, see Ref. 31]).

The simplest way to connect the truly massless cross sections in the $\overline{\mathrm{MS}}$ scheme with the massive cross sections is to subtract the finite pieces $d_{Q \rightarrow Q}\left(x, \mu_{0}\right)$ from the massive theory. In this way, one can incorporate also the $m^{2} / p_{T}^{2}$ terms, as given in the massive theory, with the advantage that the massive theory approaches the ZM-VFNS theory in the limit $p_{T} \rightarrow \infty$ or $m \rightarrow 0$. In addition, by including also the terms proportional to $\ln m^{2}$ contained in $d_{Q \rightarrow Q}(x, \mu)$ one can obtain not only the finite subtraction terms but also the terms needed for a transition to a new factorization scale. This approach has been applied to $\gamma+\gamma \rightarrow D^{\star}+X$ [11, 32], to $\gamma+p \rightarrow D^{\star}+X$ [33] and to $p+\bar{p} \rightarrow D^{\star}+X$ 12. In particular in Ref. [12, we obtained the finite subtraction terms by comparing the cross sections of the massive theory, worked out by Bojak and Stratmann [34, 35], in the limit $m \rightarrow 0$ with the cross sections in the genuine massless theory in the $\overline{\mathrm{MS}}$ factorization scheme as deduced by Aversa et al. 36] in a form which is equivalent to the convolution of the massless cross section with $d_{Q \rightarrow Q}(x, \mu)$.

We are going to present details of this quite involved calculation in this paper. The purpose is, on the one hand, to exactly demonstrate that all the subtraction terms are generated by the convolution with partonic FFs, at NLO just with $d_{Q \rightarrow Q}(x, \mu)$. On the other hand, we hope that the detailed presentation will show how the calculation carries over to other processes $a+b \rightarrow D^{\star}+X$. Since heavy-quark production in hadron-hadron collisions is the most complex case, we shall concentrate on this particular process. Some results will also be directly relevant for heavy-quark production in $\gamma \gamma$ and $\gamma p$ processes. 


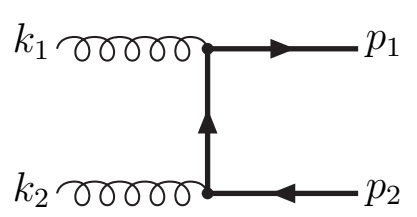

(a)

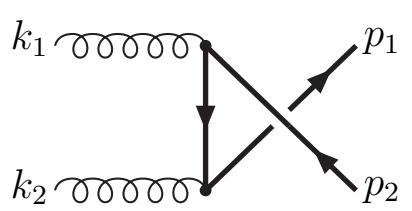

(b)

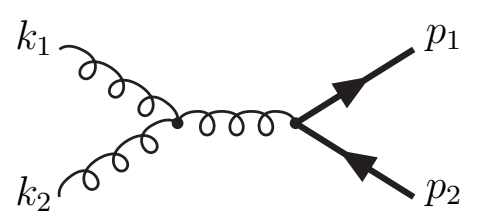

(c)

Figure 1: Feynman diagrams for the LO gluon-gluon fusion process $g+g \rightarrow Q+\bar{Q}$.

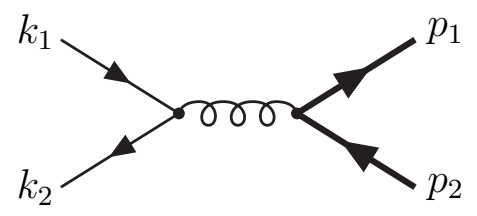

Figure 2: The LO quark-antiquark annihilation process $q+\bar{q} \rightarrow Q+\bar{Q}$.

The outline of this paper is as follows. In Sec. 2. we consider heavy-quark production in hadronic collisions, introduce the notation and review the derivation of the subtraction terms in Ref. [12. In Sec. 3, we present the convolution formulas, from which, in Sec. 4. the various subtraction terms are calculated and compared with the results in Ref. [12. Section 15 contains a summary and some concluding remarks. The subprocess cross sections needed in the convolutions have been collected in App. $\mathrm{A}$ for convenience.

\section{Hadroproduction of heavy quarks}

In the FFNS, the following partonic subprocesses contribute to $p+\bar{p} \rightarrow H+X$ in leading order (LO) and NLO, where $H=D, D^{\star}, B \ldots$ is a heavy meson:

1. $g\left(k_{1}\right)+g\left(k_{2}\right) \rightarrow Q\left(p_{1}\right)+\bar{Q}\left(p_{2}\right)+\left[g\left(p_{3}\right)\right]$, where $Q=c, b$ denotes a heavy quark. The LO Feynman diagrams are shown in Fig. 团.

2. $q\left(k_{1}\right)+\bar{q}\left(k_{2}\right) \rightarrow Q\left(p_{1}\right)+\bar{Q}\left(p_{2}\right)+\left[g\left(p_{3}\right)\right]$. In LO, there is one Feynman diagram, which is shown in Fig. 2 .

3. $g\left(k_{1}\right)+q\left(k_{2}\right) \rightarrow Q\left(p_{1}\right)+\bar{Q}\left(p_{2}\right)+q\left(p_{3}\right)$ and $g\left(k_{1}\right)+\bar{q}\left(k_{2}\right) \rightarrow Q\left(p_{1}\right)+\bar{Q}\left(p_{2}\right)+\bar{q}\left(p_{3}\right)$ contribute at NLO. The Feynman diagrams for these processes, as well as those for the NLO contributions of $g g \rightarrow Q \bar{Q} g$ and $q \bar{q} \rightarrow Q \bar{Q} g$, can be found in App. B. 
Our aim is to calculate differential cross sections with an observed heavy quark $Q$ of momentum $p_{1}$. Therefore we define the following invariants

$$
\begin{aligned}
s & =\left(k_{1}+k_{2}\right)^{2}, \\
t_{1} & =t-m^{2}=\left(k_{1}-p_{1}\right)^{2}-m^{2}, \\
u_{1} & =u-m^{2}=\left(k_{2}-p_{1}\right)^{2}-m^{2},
\end{aligned}
$$

and

$$
s_{2}=S_{2}-m^{2}=\left(k_{1}+k_{2}-p_{1}\right)^{2}-m^{2}=s+t_{1}+u_{1}
$$

with $s+t_{1}+u_{1}=0$ at LO, where $p_{3}=0$. As usual, we introduce the dimensionless variables $v$ and $w$ by

$$
v=1+\frac{t_{1}}{s}, \quad w=-\frac{u_{1}}{s+t_{1}}
$$

so that

$$
t_{1}=-s(1-v), \quad u_{1}=-s v w
$$

In LO, we have $w=1$.

In a recent publication [12, we have presented a NLO calculation for the inclusive production of $D^{\star}$ mesons in $p \bar{p}$ collisions including heavy-quark mass effects in the hard-scattering cross sections. The following procedure has been adopted [11, 32] (see also Refs. [7, 37]):

(i) We have derived the zero-mass limit of the cross sections in the massive FFNS with $n_{f}=3$ [34, 35] for the partonic subprocesses listed above only keeping $m$ as a regulator in logarithms $\ln \left(\mathrm{m}^{2} / \mathrm{s}\right)$. Special care was required in order to recover the distributions $\delta(1-w),(1 /(1-w))_{+}$and $(\ln (1-w) /(1-w))_{+}$occurring in the massless $\overline{\mathrm{MS}}$ calculation, see, e.g., Eq. (12) in Ref. [12]. The result, generically denoted $\lim _{m \rightarrow 0} \mathrm{~d} \tilde{\sigma}(m)$, contains mass singular logarithms $\ln \left(m^{2}\right)$, but collinear singularities associated with light quarks and gluons are already subtracted in $\mathrm{d} \tilde{\sigma}(m)$.

(ii) Then we have compared the massless limit with the corresponding hard-scattering cross sections in the genuine massless $\overline{\mathrm{MS}}$ calculation in order to identify appropriate subtraction terms. Generically, one can write

$$
\mathrm{d} \sigma^{\mathrm{sub}}=\lim _{m \rightarrow 0} \mathrm{~d} \tilde{\sigma}(m)-\mathrm{d} \hat{\sigma}_{\overline{\mathrm{MS}}},
$$

where $\mathrm{d} \hat{\sigma}_{\overline{\mathrm{MS}}}$ is a hard-scattering cross section in the genuine $\overline{\mathrm{MS}}$ calculation.

(iii) The desired massive hard-scattering cross sections have then been constructed by removing the subtraction terms from the massive cross sections in the fixed-order theory:

$$
\mathrm{d} \hat{\sigma}(m)=\mathrm{d} \tilde{\sigma}(m)-\mathrm{d} \sigma^{\mathrm{sub}} .
$$

By this procedure, the collinear logarithms $\ln \left(\mathrm{m}^{2} / \mathrm{s}\right)$ along with finite terms which are independent of the heavy-quark mass are subtracted from $\mathrm{d} \tilde{\sigma}(m)$. On the other hand, all finite mass terms of the form $\left(m^{2} / p_{T}^{2}\right)^{n}$ (with an integer $n$ ) are kept in the hard-scattering cross sections. 
(iv) Contributions with charm quarks in the initial state have been included in the massless approach. It can be shown that neglecting the corresponding heavy-quark mass terms corresponds to a convenient choice of scheme (S-ACOT scheme 38) which does not imply any loss of precision. In fact, the error which is made is of the same order as the error of the factorization formula, as has been discussed in the context of heavy-quark production in deep inelastic scattering [38, 39]. Obviously, this rule is of great practical importance since the existing massless results for the hardscattering cross sections [36] can simply be used, whereas their massive analogues are unknown and would require a dedicated calculation of these processes. ${ }^{3}$

Note that also the FONLL calculation in Ref. [21] has been constructed with the help of the zero-mass limit of the fixed-order calculation in Refs. 1, 2. On the other hand, in the GM-VFNS of Ref. [29], the collinear subtractions have been obtained using the methods of mass factorization in a massive regularization scheme. In this approach, the subtraction terms are computed by convolutions of appropriate subprocesses with universal partonic PDFs and FFs. However, the discussion in Ref. 29] is rather generic without presenting many details. It is the purpose of this paper to provide a detailed description of the derivation of the collinear subtraction terms using the convolution method and to compare with the results obtained in our previous publication [12].

\section{Mass factorization with massive quarks}

The starting point in our approach is the basic factorization formula at the partonic level:

$$
\mathrm{d} \tilde{\sigma}(a+b \rightarrow Q+X)=f_{a \rightarrow i}\left(x_{1}\right) \otimes f_{b \rightarrow j}\left(x_{2}\right) \otimes \mathrm{d} \hat{\sigma}(i+j \rightarrow k+X) \otimes d_{k \rightarrow Q}(z)
$$

where d $\tilde{\sigma}$ denote partonic cross sections (with singularities due to light-quark and gluon lines already subtracted via conventional mass factorization [40]) and d $\hat{\sigma}$ are IR-safe hardscattering cross sections which are free of logarithms of the heavy-quark mass. The indices $a, b$, and $i, j, k$ denote partons, and a sum over double indices is implied here and in the following. All logarithms of the heavy-quark mass (i.e. the mass singularities in the zero-mass limit) are contained in the partonic distribution functions $f_{a \rightarrow i}$ and in the partonic fragmentation functions $d_{k \rightarrow Q}$. The convolution $\otimes$ denotes the usual convolution integral and will be specified below in Eqs. (14), (23) and (30). Equation (17) reflects the fact that the partonic cross sections $\mathrm{d} \tilde{\sigma}$ can be factorized into process-dependent IR-safe hard-scattering cross sections $\mathrm{d} \hat{\sigma}$, which are well-behaved and finite in the limit $m \rightarrow 0$, and universal (process-independent) partonic PDFs $f_{a \rightarrow i}$ and partonic FFs $d_{k \rightarrow Q}$.

\footnotetext{
${ }^{3}$ For deep inelastic scattering, massive-quark-initiated coefficients have been obtained in Refs. 9, 10; the results for this simpler case are already quite involved.
} 
Equation (17) can be expanded in powers of $\alpha_{s}$ with the help of

$$
\begin{aligned}
f_{a \rightarrow i}\left(x_{1}\right) & =\delta_{i a} \delta\left(1-x_{1}\right)+f_{a \rightarrow i}^{(1)}+f_{a \rightarrow i}^{(2)}+\ldots \\
f_{b \rightarrow j}\left(x_{2}\right) & =\delta_{j b} \delta\left(1-x_{2}\right)+f_{b \rightarrow j}^{(1)}+f_{b \rightarrow j}^{(2)}+\ldots \\
d_{k \rightarrow Q}(z) & =\delta_{k Q} \delta(1-z)+d_{k \rightarrow Q}^{(1)}+d_{k \rightarrow Q}^{(2)}+\ldots, \\
\mathrm{d} \hat{\sigma} & =\mathrm{d} \hat{\sigma}^{(0)}+\mathrm{d} \hat{\sigma}^{(1)}+\mathrm{d} \hat{\sigma}^{(2)}+\ldots \\
\mathrm{d} \tilde{\sigma} & =\mathrm{d} \tilde{\sigma}^{(0)}+\mathrm{d} \tilde{\sigma}^{(1)}+\mathrm{d} \tilde{\sigma}^{(2)}+\ldots
\end{aligned}
$$

For the partonic PDFs and FFs, the superscript denotes the order of $\alpha_{s}$. For the cross sections, it indicates the relative order in $\alpha_{s}$ with respect to the Born cross sections. The expansion of Eq. (77) can be used to determine order by order the relation between the hard-scattering and partonic cross sections. Up to NLO, one finds

$$
\begin{aligned}
\mathrm{d} \hat{\sigma}^{(0)}(a+b \rightarrow Q+X)= & \mathrm{d} \tilde{\sigma}^{(0)}(a+b \rightarrow Q+X)=\mathrm{d} \sigma^{(0)}(a+b \rightarrow Q+X) \\
\mathrm{d} \hat{\sigma}^{(1)}(a+b \rightarrow Q+X)= & \mathrm{d} \tilde{\sigma}^{(1)}(a+b \rightarrow Q+X) \\
& -f_{a \rightarrow i}^{(1)}\left(x_{1}\right) \otimes \mathrm{d} \sigma^{(0)}(i+b \rightarrow Q+X) \\
& -f_{b \rightarrow j}^{(1)}\left(x_{2}\right) \otimes \mathrm{d} \sigma^{(0)}(a+j \rightarrow Q+X) \\
& -\mathrm{d} \sigma^{(0)}(a+b \rightarrow k+X) \otimes d_{k \rightarrow Q}^{(1)}(z)
\end{aligned}
$$

The three convolutions in Eq. (10) can be identified with the subtraction term $\mathrm{d} \sigma^{\text {sub }}$ in Eq. (6).

The factorization in Eq. (77) has to be defined at a definite energy or momentum scale which enters as an argument into the PDFs, FFs and $\mathrm{d} \hat{\sigma}$. We denote the factorization scales by $\mu_{F}$ for initial-state factorization (entering the PDFs) and by $\mu_{F}^{\prime}$ for final-state factorization (entering the FFs). The renormalization scale will be called $\mu_{R}$.

\subsection{Partonic parton distribution and fragmentation functions}

The functions $f_{i \rightarrow j}^{(1)}$ for the initial state are given in the $\overline{\mathrm{MS}} \mathrm{scheme}^{4}$, keeping the heavyquark mass as a regulator for the collinear divergences, by

$$
\begin{aligned}
f_{g \rightarrow Q}^{(1)}\left(x, \mu_{R}, \mu_{F}\right) & =\frac{\alpha_{s}\left(\mu_{R}\right)}{2 \pi} P_{g \rightarrow q}^{(0)}(x) \ln \frac{\mu_{F}^{2}}{m^{2}}, \\
f_{Q \rightarrow Q}^{(1)}\left(x, \mu_{R}, \mu_{F}\right) & =\frac{\alpha_{s}\left(\mu_{R}\right)}{2 \pi} C_{F}\left[\frac{1+x^{2}}{1-x}\left(\ln \frac{\mu_{F}^{2}}{m^{2}}-2 \ln (1-x)-1\right)\right]_{+}, \\
f_{g \rightarrow g}^{(1)}\left(x, \mu_{R}, \mu_{F}\right) & =-\frac{\alpha_{s}\left(\mu_{R}\right)}{2 \pi} \frac{2}{3} T_{f} \ln \frac{\mu_{F}^{2}}{m^{2}} \delta(1-x),
\end{aligned}
$$

\footnotetext{
${ }^{4}$ Note that it is assumed that the $\overline{\mathrm{MS}}$ scheme is defined in the conventional way where photons and gluons have $d-2$ degrees of freedom (where $d$ is the number of space-time dimensions). Furthermore, subtractions $f_{i j} \otimes \mathrm{d} \sigma^{(0)}$ are performed with subprocess cross sections calculated in $d$ dimensions.
} 
where $P_{g \rightarrow q}^{(0)}(x)=\frac{1}{2}\left[x^{2}+(1-x)^{2}\right]$ and $P_{q \rightarrow q}^{(0)}(x)=C_{F}\left[\left(1+x^{2}\right) /(1-x)\right]_{+}$(appearing in $\left.f_{Q \rightarrow Q}^{(1)}\right)$ are the conventional (space-like) one-loop splitting functions and $T_{f}=1 / 2$. The function $f_{Q \rightarrow Q}^{(1)}\left(x, \mu_{R}, \mu_{F}\right)$ will not be used in the following, since heavy quarks in the initial state are treated as massless quarks as explained in Sec. 2. It would be present in cases where massive heavy quarks $Q$ appear in the initial state as for example in Refs. [9, 10, 26, 27.

The functions $d_{i \rightarrow j}^{(1)}$ for the final state read [8, 9, 10, 14]

$$
\begin{aligned}
d_{g \rightarrow Q}^{(1)}\left(z, \mu_{R}, \mu_{F}^{\prime}\right) & =\frac{\alpha_{s}\left(\mu_{R}\right)}{2 \pi} P_{g \rightarrow q}^{(0)}(z) \ln \frac{\mu_{F}^{\prime 2}}{m^{2}} \\
d_{Q \rightarrow Q}^{(1)}\left(z, \mu_{R}, \mu_{F}^{\prime}\right) & =\frac{\alpha_{s}\left(\mu_{R}\right)}{2 \pi} C_{F}\left[\frac{1+z^{2}}{1-z}\left(\ln \frac{\mu_{F}^{\prime 2}}{m^{2}}-2 \ln (1-z)-1\right)\right]_{+} .
\end{aligned}
$$

Generally, the splitting functions entering the partonic FFs are time-like splitting functions which are, however, identical to the space-like splitting functions at the one-loop level. It should be noted that the function $f_{Q \rightarrow Q}^{(1)}\left(x, \mu_{R}, \mu_{F}\right)$ in Eq. (11) is of the same form as $d_{Q \rightarrow Q}^{(1)}\left(x, \mu_{R}, \mu_{F}^{\prime}\right)$ at $\mathcal{O}\left(\alpha_{s}^{1}\right)[9$, 10. This will not be true at higher orders since the NLO space- and time-like splitting functions $P_{q \rightarrow q}^{(1)}(x)$ are different. All the other partonic PDFs and FFs not listed here are zero at $\mathcal{O}\left(\alpha_{s}^{1}\right)$. Furthermore, analogous results for processes involving photon splittings can be found by obvious replacements $\left(g \rightarrow \gamma, \alpha_{s} \rightarrow \alpha\right.$ and appropriate modifications of colour factors) in Eqs. (11) and (12).

The partonic PDFs and FFs are known to order $\mathcal{O}\left(\alpha_{s}^{2}\right)$. They would be needed, together with the three-loop beta function of QCD, for computing subtraction terms at next-toNLO (NNLO). For the initial state, the partonic PDFs at order $\mathcal{O}\left(\alpha_{s}^{2}\right)$ can be found in Ref. [41] (with the exception of $f_{Q \rightarrow Q}^{(2)}(x)$, which is unknown). Recently, also the $\mathcal{O}\left(\alpha_{s}^{2}\right)$ contributions to the perturbative FFs have been derived [15, 16]. It should be noted that, at $\mathcal{O}\left(\alpha_{s}^{2}\right)$, all of the perturbative PDFs and FFs are non-vanishing at $\mu_{F}=m$ and $\mu_{F}^{\prime}=m$, respectively. In fact, the parts proportional to logarithms of the factorization scale follow from the evolution equations, so that the new information obtained from the $\mathcal{O}\left(\alpha_{s}^{2}\right)$ calculation is contained in the non-vanishing pieces at $\mu_{F}, \mu_{F}^{\prime}=m$.

In Sec. 4, we will need the distribution $d_{Q \rightarrow Q}^{(1)}(\bar{z})$ with $\bar{z}=1-v+v w$ as a distribution in the kinematic variables $v$ and $w$. This form of $d_{Q \rightarrow Q}^{(1)}(\bar{z})$ can be written as:

$$
d_{Q \rightarrow Q}^{(1)}(\bar{z})=A(v) \delta(1-w)+B(v) \frac{1}{(1-w)_{+}}+C(v)\left(\frac{\ln (1-w)}{(1-w)}\right)_{+}+D(v, w)
$$




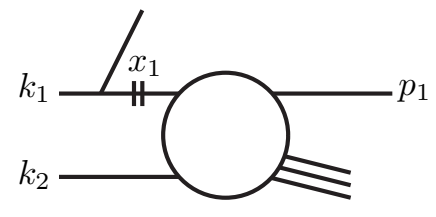

(a)

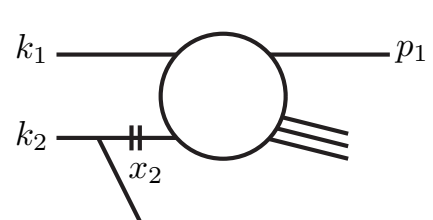

$(b)$

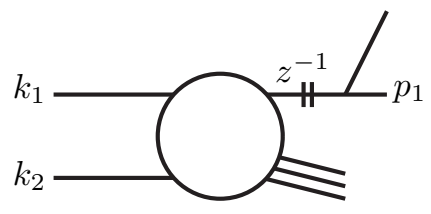

(c)

Figure 3: Sketch of kinematics of mass factorization for (a) upper incoming line (b) lower incoming line and (c) outgoing line.

with

$$
\begin{aligned}
A(v) & =C_{F} \frac{\alpha_{s}\left(\mu_{R}\right)}{2 \pi} \frac{1}{2 v}\left[\ln \frac{\mu_{F}^{\prime 2}}{m^{2}}(3+4 \ln v)+4\left(1-\ln v-\ln ^{2} v\right)\right], \\
B(v) & =C_{F} \frac{\alpha_{s}\left(\mu_{R}\right)}{2 \pi} \frac{2}{v}\left[\ln \frac{\mu_{F}^{\prime 2}}{m^{2}}-1-2 \ln v\right], \\
C(v) & =-C_{F} \frac{\alpha_{s}\left(\mu_{R}\right)}{2 \pi} \frac{4}{v}, \\
D(v, w) & =-C_{F} \frac{\alpha_{s}\left(\mu_{R}\right)}{2 \pi}[2-v(1-w)]\left[\ln \frac{\mu_{F}^{\prime 2}}{m^{2}}-1-2 \ln v-2 \ln (1-w)\right] .
\end{aligned}
$$

\subsection{Subtraction terms at NLO}

We distinguish mass factorization in the initial state and in the final state. For one-particle inclusive production, where one final-state particle has a fixed momentum (above, we had chosen $p_{1}$ ), we have to distinguish further two cases with initial-state singularities corresponding to $t$ - and $u$-channel scattering. A graphical representation of the subtraction terms in form of cut diagrams for all cases is shown in Fig. 3. These diagrams can be found by applying all possible cuts to internal lines of the Feynman diagrams (see App. B). The cuts are allowed if the $2 \rightarrow 2$ subprocesses are kinematically possible and the $1 \rightarrow 2$ process involves the splitting into a heavy-quark line. In an axial gauge, the cut diagrams correspond to actual Feynman diagrams.

\subsubsection{Initial-state factorization}

In the first case with $u$-channel scattering (see Fig. 3(a)), the collinear subtractions are given by

$$
\begin{aligned}
\mathrm{d} \sigma^{\mathrm{sub}}(a+b \rightarrow Q+X) & =\int_{0}^{1} \mathrm{~d} x_{1} f_{a \rightarrow i}^{(1)}\left(x_{1}, \mu_{R}, \mu_{F}\right) \mathrm{d} \hat{\sigma}^{(0)}\left(i\left(x_{1} k_{1}\right)+b\left(k_{2}\right) \rightarrow Q\left(p_{1}\right)+X\right) \\
& \equiv f_{a \rightarrow i}^{(1)}\left(x_{1}\right) \otimes \mathrm{d} \hat{\sigma}^{(0)}(i+b \rightarrow Q+X)
\end{aligned}
$$


Here $a+b \rightarrow Q+X$ stands for the one-particle inclusive partonic subprocesses $(g+g \rightarrow$ $Q+X, q+\bar{q} \rightarrow Q+X, g+q \rightarrow Q+X, g+\bar{q} \rightarrow Q+X), f_{a \rightarrow i}\left(x_{1}, \mu_{R}, \mu_{F}\right)$ describes the collinear splitting of parton ' $a$ ' into parton ' $i$ ', and $i+b \rightarrow Q+X$ are the corresponding $2 \rightarrow 2$ subprocesses with momenta $x_{1} k_{1}, k_{2}$ and $p_{1}$. A sum over $i$ is implied, i.e., all possible splittings and subprocesses have to be taken into account.

We define the following invariants for the subprocess:

$$
\begin{aligned}
\hat{s} & =\left(x_{1} k_{1}+k_{2}\right)^{2}=x_{1} s, \\
\hat{t}_{1} & =\left(x_{1} k_{1}-p_{1}\right)^{2}-m^{2}=x_{1} t_{1}, \\
\hat{u}_{1} & =\left(k_{2}-p_{1}\right)^{2}-m^{2}=u_{1},
\end{aligned}
$$

and

$$
\begin{array}{ll}
\hat{v}=1+\frac{\hat{t}_{1}}{\hat{s}}=v \quad, \quad \hat{w}=-\frac{\hat{u}_{1}}{\hat{s}+\hat{t}_{1}}=\frac{w}{x_{1}}, \\
\hat{t}_{1}=-\hat{s}(1-\hat{v}) \quad, \quad \hat{u}_{1}=-\hat{s} \hat{v} \hat{w}=-\hat{s} \hat{v} .
\end{array}
$$

For the calculation of $\mathrm{d}^{2} \sigma^{\text {sub }} /(\mathrm{d} v \mathrm{~d} w)$ in Eq. (14) , it is convenient to write the subprocess cross section as

$$
\frac{\mathrm{d}^{2} \hat{\sigma}^{(0)}}{\mathrm{d} v \mathrm{~d} w}=J \frac{\mathrm{d}^{2} \hat{\sigma}^{(0)}}{\mathrm{d} \hat{v} \mathrm{~d} \hat{w}}=J \frac{\mathrm{d} \hat{\sigma}^{(0)}}{\mathrm{d} \hat{v}} \delta(1-\hat{w})
$$

with

$$
\delta(1-\hat{w})=w \delta\left(x_{1}-\bar{x}_{1}\right), \quad \bar{x}_{1}=w .
$$

The $\delta$-function imposes the $2 \rightarrow 2$ process kinematics $\hat{s}+\hat{t}_{1}+\hat{u}_{1}=0$, i.e. $\hat{w}=1$, and implies $\bar{x}_{1}=w$. The Jacobian reads

$$
J=\frac{\partial(\hat{v}, \hat{w})}{\partial(v, w)}=\frac{1}{x_{1}} .
$$

Combining these results we find

$$
\frac{\mathrm{d}^{2} \hat{\sigma}^{(0)}}{\mathrm{d} v \mathrm{~d} w}=\left.\frac{\mathrm{d} \hat{\sigma}^{(0)}}{\mathrm{d} \hat{v}}\right|_{\hat{s} \rightarrow \bar{x}_{1} s, \hat{v} \rightarrow v} \delta\left(x_{1}-\bar{x}_{1}\right),
$$

so that the subtraction terms for initial-state factorization of the upper incoming line can be calculated as

$$
\frac{\mathrm{d}^{2} \sigma^{\mathrm{sub}}}{\mathrm{d} v \mathrm{~d} w}(a+b \rightarrow Q+X)=\left.f_{a \rightarrow i}^{(1)}\left(\bar{x}_{1}, \mu_{R}, \mu_{F}\right) \frac{\mathrm{d} \hat{\sigma}^{(0)}}{\mathrm{d} \hat{v}}(i+b \rightarrow Q+X)\right|_{\hat{v} \rightarrow v, \hat{s} \rightarrow \bar{x}_{1} s} .
$$

In the second case with $t$-channel scattering (see Fig. 3(b)), the collinear subtractions are given by

$$
\begin{aligned}
\mathrm{d} \sigma^{\mathrm{sub}}(a+b \rightarrow Q+X) & =\int_{0}^{1} \mathrm{~d} x_{2} f_{b \rightarrow j}^{(1)}\left(x_{2}, \mu_{R}, \mu_{F}\right) \mathrm{d} \hat{\sigma}^{(0)}\left(a\left(k_{1}\right)+j\left(x_{2} k_{2}\right) \rightarrow Q\left(p_{1}\right)+X\right) \\
& \equiv f_{b \rightarrow j}^{(1)}\left(x_{2}\right) \otimes \mathrm{d} \hat{\sigma}^{(0)}(a+j \rightarrow Q+X)
\end{aligned}
$$


The invariants for the subprocess are now given by

$$
\begin{aligned}
\hat{s} & =\left(k_{1}+x_{2} k_{2}\right)^{2}=x_{2} s, \\
\hat{t}_{1} & =\left(k_{1}-p_{1}\right)^{2}-m^{2}=t_{1}, \\
\hat{u}_{1} & =\left(x_{2} k_{2}-p_{1}\right)^{2}-m^{2}=x_{2} u_{1},
\end{aligned}
$$

and

$$
\hat{v}=\frac{x_{2}-1+v}{x_{2}}, \quad \hat{w}=\frac{x_{2} v w}{x_{2}-1+v} .
$$

Again, we write the subprocess cross section as in Eq. (18) with

$$
\delta(1-\hat{w})=\bar{x}_{2}^{2} \frac{v w}{1-v} \delta\left(x_{2}-\bar{x}_{2}\right), \quad \bar{x}_{2}=\frac{1-v}{1-v w},
$$

and

$$
J=\frac{\partial(\hat{v}, \hat{w})}{\partial(v, w)}=\frac{v}{x_{2}-1+v} .
$$

For the $2 \rightarrow 2$ subprocess kinematics, we have $\hat{w}=1, x_{2}=\bar{x}_{2}, \hat{v}=v w$ and $J=1 /\left(\bar{x}_{2} w\right)$. Combining these results, we find

$$
\frac{\mathrm{d}^{2} \hat{\sigma}^{(0)}}{\mathrm{d} v \mathrm{~d} w}=\left.\frac{v}{1-v w} \frac{\mathrm{d} \hat{\sigma}^{(0)}}{\mathrm{d} \hat{v}}\right|_{\hat{s} \rightarrow \bar{x}_{2} s, \hat{v} \rightarrow v w} \delta\left(x_{2}-\bar{x}_{2}\right)
$$

so that the subtraction terms for initial-state factorization of the lower incoming line can be calculated as

$$
\frac{\mathrm{d}^{2} \sigma^{\mathrm{sub}}}{\mathrm{d} v \mathrm{~d} w}(a+b \rightarrow Q+X)=\left.f_{b \rightarrow j}^{(1)}\left(\bar{x}_{2}, \mu_{R}, \mu_{F}\right) \frac{v}{1-v w} \frac{\mathrm{d} \hat{\sigma}^{(0)}}{\mathrm{d} \hat{v}}(a+j \rightarrow Q+X)\right|_{\hat{v} \rightarrow v w, \hat{s} \rightarrow \bar{x}_{2} s}
$$

\subsubsection{Final-state factorization}

The case shown in Fig. 3(c) corresponds to factorization of singularities in the final state. Here the collinear subtractions are given by

$$
\begin{aligned}
\mathrm{d} \sigma^{\mathrm{sub}}(a+b \rightarrow Q+X) & =\int_{0}^{1} \mathrm{~d} z \mathrm{~d} \hat{\sigma}^{(0)}\left(a\left(k_{1}\right)+b\left(k_{2}\right) \rightarrow k\left(z^{-1} p_{1}\right)+X\right) d_{k \rightarrow Q}^{(1)}\left(z, \mu_{R}, \mu_{F}^{\prime}\right) \\
& \equiv \mathrm{d} \hat{\sigma}^{(0)}(a+b \rightarrow k+X) \otimes d_{k \rightarrow Q}^{(1)}(z)
\end{aligned}
$$

The invariants for the subprocess can be defined as follows

$$
\begin{aligned}
\hat{s} & =\left(k_{1}+k_{2}\right)^{2}=s, \\
\hat{t}_{1} & =\left(k_{1}-z^{-1} p_{1}\right)^{2}-m^{2}=\frac{1}{z} t_{1}, \\
\hat{u}_{1} & =\left(k_{2}-z^{-1} p_{1}\right)^{2}-m^{2}=\frac{1}{z} u_{1},
\end{aligned}
$$


and

$$
\hat{v}=\frac{z-1+v}{z}, \quad \hat{w}=\frac{v w}{z-1+v} .
$$

As before, we write the subprocess cross section as in Eq. (18) with

$$
\delta(1-\hat{w})=v w \delta(z-\bar{z}), \quad \bar{z}=1-v+v w,
$$

and

$$
J=\frac{\partial(\hat{v}, \hat{w})}{\partial(v, w)}=\frac{1}{z} \frac{v}{z-1+v} .
$$

From $\hat{w}=1$ one finds $\bar{z}=1-v+v w, \hat{v}=v w / \bar{z}$ and $J=1 /(\bar{z} w)$. Combining these results, we find

$$
\frac{\mathrm{d}^{2} \hat{\sigma}^{(0)}}{\mathrm{d} v \mathrm{~d} w}=\left.\frac{v}{\bar{z}} \frac{\mathrm{d} \hat{\sigma}^{(0)}}{\mathrm{d} \hat{v}}\right|_{\hat{s} \rightarrow s, \hat{v} \rightarrow \frac{v w}{\bar{z}}} \delta(z-\bar{z}),
$$

so that the subtraction terms for final-state factorization can be calculated as

$$
\frac{\mathrm{d}^{2} \sigma^{\mathrm{sub}}}{\mathrm{d} v \mathrm{~d} w}(a+b \rightarrow Q+X)=\left.d_{k \rightarrow Q}^{(1)}\left(\bar{z}, \mu_{R}, \mu_{F}^{\prime}\right) \frac{v}{\bar{z}} \frac{\mathrm{d} \hat{\sigma}^{(0)}}{\mathrm{d} \hat{v}}(a+b \rightarrow k+X)\right|_{\hat{v} \rightarrow v w / \bar{z}, \hat{s} \rightarrow s}
$$

\subsection{Scheme dependence and implementation freedom}

Before we turn to a discussion of our results for the collinear subtraction terms calculated according to Eqs. (22), (29) and (36), we add some additional remarks.

The partonic PDFs and FFs introduced in Sec. 3.1 are given in the $\overline{\mathrm{MS}}$ factorization and renormalization scheme. However, in the FFNS calculations of heavy-quark production 1. 2, 3, 4, 34, 35, a modification of the $\overline{\mathrm{MS}}$ scheme has been adopted, called $\overline{\mathrm{MS}}_{m}$ or decoupling scheme 42, where divergences due to light quarks and gluons are treated in the $\overline{\mathrm{MS}}$ scheme and divergences arising from heavy-quark loops are subtracted at zero

momentum. In order to switch from the $\overline{\mathrm{MS}}_{m}$ to the $\overline{\mathrm{MS}}$ scheme the following terms have to be added to the partonic cross sections of the fixed-order calculations (see Sec. 3 in Ref. [21]):

$$
\begin{array}{ll}
-\alpha_{s}\left(\mu_{R}\right) \frac{2 T_{f}}{3 \pi} \ln \frac{\mu_{R}^{2}}{m^{2}} \mathrm{~d} \sigma_{q \bar{q}}^{(0)} & (q+\bar{q} \rightarrow Q+X), \\
-\alpha_{s}\left(\mu_{R}\right) \frac{2 T_{f}}{3 \pi} \ln \frac{\mu_{R}^{2}}{\mu_{F}^{2}} \mathrm{~d} \sigma_{g g}^{(0)} & (g+g \rightarrow Q+X) .
\end{array}
$$

In Eqs. (37) and (38), the parts proportional to $\ln \mu_{R}^{2}$ are due to the change of $\alpha_{s}$ when going from the $\overline{\mathrm{MS}}_{m}$ to the $\overline{\mathrm{MS}}$ scheme. If $\alpha_{s}^{\left(n_{f}-1\right)}\left(\mu_{R}\right)$ and $\alpha_{s}^{\left(n_{f}\right)}\left(\mu_{R}\right)$ denote the strongcoupling constants in the $\overline{\mathrm{MS}}_{m}$ and $\overline{\mathrm{MS}}$ schemes, respectively, one can derive from the renormalization group equation the following relationship between the couplings:

$$
\alpha_{s}^{\left(n_{f}-1\right)}\left(\mu_{R}\right)=\alpha_{s}^{\left(n_{f}\right)}\left(\mu_{R}\right)\left(1-\frac{\alpha_{s}^{\left(n_{f}\right)}\left(\mu_{R}\right)}{3 \pi} T_{f} \ln \frac{\mu_{R}^{2}}{m^{2}}\right)+\mathcal{O}\left(\alpha_{s}^{3}\right)
$$




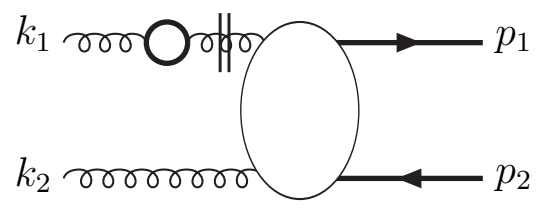

(a)

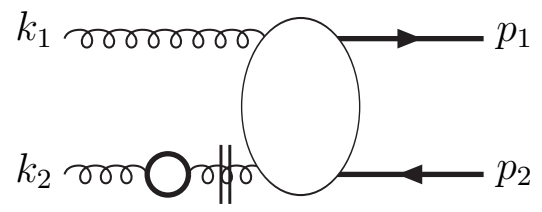

(b)

Figure 4: Feynman diagrams representing (a) $f_{g \rightarrow g}^{(1)}\left(x_{1}\right) \otimes \mathrm{d} \hat{\sigma}^{(0)}(g g \rightarrow Q \bar{Q})$ and (b) $f_{g \rightarrow g}^{(1)}\left(x_{2}\right) \otimes \mathrm{d} \hat{\sigma}^{(0)}(g g \rightarrow Q \bar{Q})$. The fermion loops on the external gluon lines are heavy-quark loops.

The parts proportional to $\ln \mu_{F}^{2}$ can be obtained by subtracting from the cross section in the gluon-gluon channel the term $\left(f_{g \rightarrow g}^{(1)}\left(x_{1}\right)+f_{g \rightarrow g}^{(1)}\left(x_{2}\right)\right) \otimes \mathrm{d} \hat{\sigma}^{(0)}(g+g \rightarrow Q+\bar{Q})$ (see Fig. (4). Since the function $f_{g \rightarrow g}^{(1)}(x)$ in Eq. (11) is proportional to Dirac's delta function, this amounts to a simple multiplication with the Born cross section in the gluon-gluon channel. This subtraction term takes into account the different treatment of heavy-quark loop contributions to external gluon lines in the $\overline{\mathrm{MS}}$ and the $\overline{\mathrm{MS}}_{m}$ schemes. The coefficients in Ref. [12 are given in the $\overline{\mathrm{MS}}_{m}$ scheme. Changing the results in Ref. [12] to the $\overline{\mathrm{MS}}$ scheme according to Eqs. (37) and (38) has the expected effect of replacing $\beta_{0}^{\left(n_{f}-1\right)}$ by $\beta_{0}^{\left(n_{f}\right)}$ in the coefficients $\hat{d}_{1}$ and $\tilde{d}_{1}$ in Eqs. (28), (29) and (55) of Ref. [12, so that in the $\overline{\mathrm{MS}}$ scheme $\Delta \hat{d}_{1}=\Delta \tilde{d}_{1}=0$ in Eqs. (35), (36) and (59) of Ref. [12.

Even fixing the factorization scheme to the $\overline{\mathrm{MS}}$ scheme leaves some freedom in the implementation of a massive VFNS, as has been discussed for the case of deep inelastic scattering in Ref. 6]. Consider for example the condition

$$
\lim _{m \rightarrow 0}\left(\mathrm{~d} \tilde{\sigma}(m)-\mathrm{d} \sigma^{\mathrm{sub}}(m)\right) \stackrel{!}{=} \mathrm{d} \hat{\sigma}_{\overline{\mathrm{MS}}}
$$

which might be used as an attempt to define the subtraction terms. d $\hat{\sigma}_{\overline{\mathrm{MS}}}$ is the massless hard-scattering cross section in the $\overline{\mathrm{MS}}$ scheme. However, this requirement fixes the subtraction term $\mathrm{d} \sigma^{\mathrm{sub}}\left(\mu / m, m / p_{T}\right)$ only up to terms $m / p_{T}$ vanishing in the limit $m \rightarrow 0$. The precise treatment of such terms proportional to $m / p_{T}$ is not prescribed by factorization. The prescription in Eq. (5), $\mathrm{d} \sigma^{\mathrm{sub}}=\lim _{m \rightarrow 0} \mathrm{~d} \tilde{\sigma}(m)-\mathrm{d} \hat{\sigma}_{\overline{\mathrm{MS}}}$, is minimal in the sense that no finite mass terms are removed from the hard part in addition to the collinear logarithms $\ln \left(m^{2} / s\right)$.

The same is true from the point of view of mass factorization: The factorization and renormalization scheme unambiguously determines the partonic PDFs and FFs. However, the convolution prescription leaves some freedom in the choice of the integration variable 
and, therefore, is only unique up to terms of the order $m / Q$ (where $Q$ is the hard scale). One example is the ACOT- $\chi$ prescription [6] in inclusive DIS, which guarantees the correct threshold behaviour of the heavy-quark-initiated contributions. Furthermore, it is possible to retain the mass terms in the subprocess cross sections entering the convolution formulas. Actually, this is done in the original ACOT scheme [26, 27].

\section{Subtraction terms: results}

We now present the results for the collinear subtraction terms, calculated using Eqs. (22), (29) and (36). The universal partonic PDFs can be found in Sec. 3.1. The required subprocess cross sections have been listed for completeness in App. A. We retain the heavy-quark mass terms in the subprocess cross sections entering the convolutions. In order to compare with our results in Ref. 12, these mass terms have to be dropped.

In order to facilitate the comparison with our previous results, we expand the subtraction cross section in the following form,

$$
\begin{aligned}
\frac{\mathrm{d}^{2} \sigma^{\mathrm{sub}}}{\mathrm{d} v \mathrm{~d} w}= & \Delta c_{1} \delta(1-w)+\Delta c_{2}\left(\frac{1}{1-w}\right)_{+}+\Delta c_{3}\left(\frac{\ln (1-w)}{1-w}\right)_{+} \\
& +\Delta c_{5} \ln v+\Delta c_{10} \ln (1-w)+\Delta c_{11}
\end{aligned}
$$

and use the abbreviations

$$
X=1-v w, \quad Y=1-v+v w, \quad v_{i}=i-v \quad(i=1,2)
$$

\subsection{Subtraction terms for $g+g \rightarrow Q+\bar{Q}+g$}

The coefficients $\Delta c_{i}$ are decomposed into an Abelian and two non-Abelian parts, following Ref. [35]:

$$
\Delta c_{i}=C(s)\left(C_{F}^{2} \Delta c_{i}^{\mathrm{qed}}+\frac{C_{A}^{2}}{4} \Delta c_{i}^{\mathrm{oq}}+\frac{1}{4} \Delta c_{i}^{\mathrm{kq}}\right)
$$

with

$$
C(s)=\frac{\alpha_{s}^{3}}{2\left(N^{2}-1\right) s} .
$$

There are four different cut diagrams contributing to the total result, which we discuss in the following.

$\mathrm{d} \hat{\sigma}^{(0)}(g g \rightarrow Q \bar{Q}) \otimes d_{Q \rightarrow Q}^{(1)}(z)$

The cut diagrams are shown in Fig. 15. The cross section $\mathrm{d} \hat{\sigma}^{(0)}(g g \rightarrow Q \bar{Q})$ is proportional 

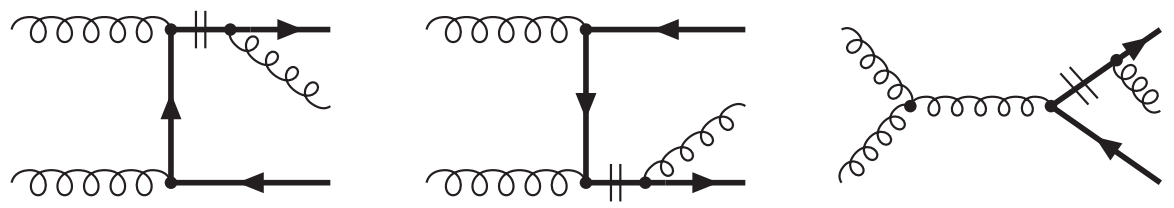

Figure 5: Feynman diagrams representing $\mathrm{d} \hat{\sigma}^{(0)}(g g \rightarrow Q \bar{Q}) \otimes d_{Q \rightarrow Q}^{(1)}(z)$.

to the function

$$
\tau(v)=\frac{v}{1-v}+\frac{1-v}{v}+\frac{4 m^{2}}{s v(1-v)}\left(1-\frac{m^{2}}{s v(1-v)}\right),
$$

which appears in the following expressions for the $\Delta c_{i}$. They are given by

$$
\begin{aligned}
& \Delta c_{1}=\left[\left(\frac{3}{4}+\ln v\right) \ln \frac{\mu_{F}^{\prime 2}}{m^{2}}+1-\ln v-\ln ^{2} v\right] \times 2 C(s) C_{F} \tau(v)\left[C_{F}-C_{A} v(1-v)\right] \\
& \Delta c_{2}=\left(\ln \frac{\mu_{F}^{\prime 2}}{m^{2}}-1-2 \ln v\right) \times 2 C(s) C_{F} \tau(v)\left[C_{F}-C_{A} v(1-v)\right] \\
& \Delta c_{3}=-2 \times 2 C(s) C_{F} \tau(v)\left[C_{F}-C_{A} v(1-v)\right] \\
& \Delta c_{5}=C(s)\left(C_{F}^{2} \Delta c_{5}^{\mathrm{qed}}+\frac{1}{4}\left(C_{A}^{2}-1\right) \Delta c_{5}^{\mathrm{oq}}\right)
\end{aligned}
$$

where

$$
\begin{aligned}
\Delta c_{5}^{\text {qed }}= & \frac{2 v}{v_{1}}-\frac{2\left(2-2 v+v^{2}\right)}{v w}+\frac{2 v^{2} w}{v_{1}}+\frac{4 v}{Y} \\
& +\frac{m^{2}}{s}\left(\frac{8 v(3-2 v)}{v_{1}}-\frac{8\left(2-2 v+v^{2}\right)}{v w}+\frac{8 v^{2} w}{v_{1}}\right) \\
+ & \frac{m^{4}}{s^{2}}\left(\frac{-8 v\left(11-15 v+6 v^{2}\right)}{v_{1}^{2}}+\frac{8 v_{1}\left(2-2 v+v^{2}\right)}{v^{2} w^{2}}\right. \\
& \left.+\frac{8\left(2+4 v-7 v^{2}+4 v^{3}\right)}{v^{2} w}+\frac{8 v^{2}(-5+4 v) w}{v_{1}^{2}}-\frac{8 v^{3} w^{2}}{v_{1}^{2}}\right) \\
\Delta c_{5}^{\mathrm{oq}}= & -4 v+\frac{8 v v_{1}^{2}}{Y^{3}}-\frac{8 v^{2} v_{1}}{Y^{2}}+\frac{4 v\left(3-6 v+4 v^{2}\right)}{Y}+\frac{m^{2}}{s}\left(-16 v+\frac{16 v}{Y}\right) \\
& +\frac{m^{4}}{s^{2}}\left(\frac{16 v(3-2 v)}{v_{1}}-\frac{16\left(2-2 v+v^{2}\right)}{v w}+\frac{16 v^{2} w}{v_{1}}\right) .
\end{aligned}
$$

We find that $\Delta c_{5}^{\mathrm{kq}}=-\Delta c_{5}^{\mathrm{oq}}$ and, finally,

$$
\begin{aligned}
& \Delta c_{10}=\Delta c_{5}, \\
& \Delta c_{11}=\frac{1}{2} \Delta c_{5}\left(1-\ln \frac{\mu_{F}^{\prime 2}}{m^{2}}\right) .
\end{aligned}
$$



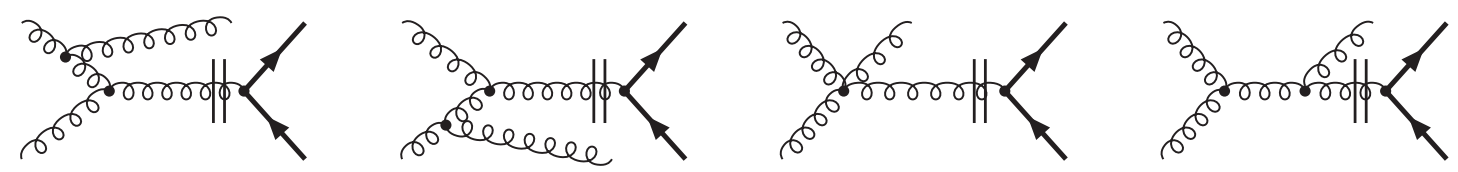

Figure 6: Feynman diagrams representing of $\mathrm{d} \hat{\sigma}^{(0)}(g g \rightarrow g g) \otimes d_{g \rightarrow Q}^{(1)}(z)$.
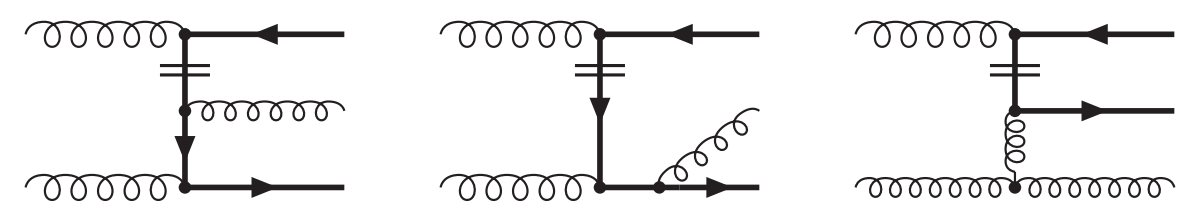

Figure 7: Feynman diagrams representing of $f_{g \rightarrow Q}^{(1)}\left(x_{1}\right) \otimes \mathrm{d} \hat{\sigma}^{(0)}(Q g \rightarrow Q g)$.

The latter two relations can be derived from $d_{Q \rightarrow Q}^{(1)}(\bar{z})$ in Eq. (13) inspecting the expressions for $B(v), C(v)$ and $D(v, w)$. (Note that the parts with $B(v)$ and $C(v)$ contribute to $\Delta c_{11}$ and $\Delta c_{10}$, respectively, in cases where the $1 /(1-w)_{+}$is canceled by factors $(1-w)$.)

Now we turn to a comparison with the results in Ref. [12, which have been derived as described in Sec. 2. For $\mu_{F}^{\prime}=m$ (and neglecting terms of the order $\mathcal{O}\left(\mathrm{m}^{2} / \mathrm{s}\right)$ ), Eqs. (46)(53) are in complete agreement with Eqs. (18) and (21)-(24) in Ref. [12. Furthermore, the parts proportional to $\ln \left(\mu_{F}^{\prime 2} / m^{2}\right)$ in Eqs. (46) and (47) are identical to Eqs. (37) and (38) in Ref. 12]. As for $\Delta c_{11}$ in Eq. (53), the part proportional to $\ln \left(\mu_{F}^{\prime 2} / m^{2}\right)$ is in agreement with Eqs. (41) and (43) for the 'qed' and 'kq' parts. The 'oq' part reproduces Eq. (42) in Ref. [12] after adding the contribution given in Eq. (54).

$\mathrm{d} \hat{\sigma}^{(0)}(g g \rightarrow g g) \otimes d_{g \rightarrow Q}^{(1)}(z)$

The cut diagram Fig. [6 only contributes to the part of $\Delta c_{11}$ proportional to $C_{A}^{2}$ :

$$
\begin{aligned}
\Delta c_{11}^{\mathrm{oq}}= & \left(-48 v^{2}+\frac{8 v_{1}\left(1-2 v+2 v^{2}\right)}{v w^{2}}+\frac{16\left(1-3 v+2 v^{2}\right)}{w}\right. \\
& +\frac{8 v^{2}\left(7-14 v+8 v^{2}\right) w}{v_{1}^{2}}-\frac{16 v^{3}(-1+2 v) w^{2}}{v_{1}^{2}}+\frac{16 v^{4} w^{3}}{v_{1}^{2}} \\
& \left.+\frac{8 v v_{1}^{2}}{Y^{3}}-\frac{8\left(3 v-5 v^{2}+2 v^{3}\right)}{Y^{2}}+\frac{8\left(7 v-6 v^{2}+2 v^{3}\right)}{Y}\right) \ln \frac{\mu_{F}^{\prime 2}}{m^{2}} .
\end{aligned}
$$

$f_{g \rightarrow Q}^{(1)}\left(x_{1}\right) \otimes \mathrm{d} \hat{\sigma}^{(0)}(Q g \rightarrow Q g)$

The $u$-channel cut in the initial state described by the diagram in Fig. 7 contributes: 

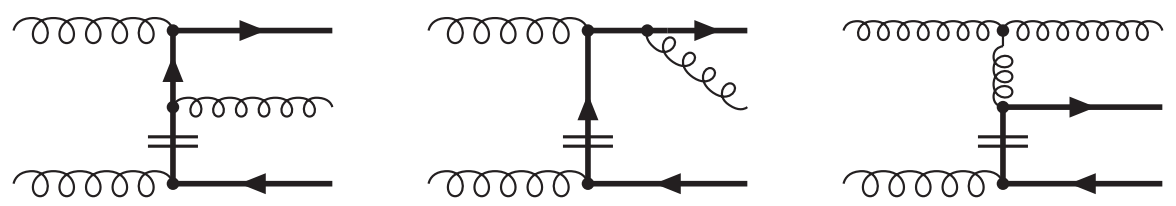

Figure 8: Feynman diagrams representing of $f_{g \rightarrow Q}^{(1)}\left(x_{2}\right) \otimes \mathrm{d} \hat{\sigma}^{(0)}(g Q \rightarrow Q g)$.

$$
\begin{aligned}
\Delta c_{11}^{\mathrm{qed}} & =\frac{1+v^{2}}{v w}\left(1-2 w+2 w^{2}\right) \ln \frac{\mu_{F}^{2}}{m^{2}}, \\
\Delta c_{11}^{\mathrm{oq}} & =\frac{1+v^{2}}{v_{1}^{2}} \frac{2}{w}\left(1-2 w+2 w^{2}\right) \ln \frac{\mu_{F}^{2}}{m^{2}}, \\
\Delta c_{11}^{\mathrm{kq}} & =-\Delta c_{11}^{\mathrm{oq}} .
\end{aligned}
$$

$f_{g \rightarrow Q}^{(1)}\left(x_{2}\right) \otimes \mathrm{d} \hat{\sigma}^{(0)}(g Q \rightarrow Q g)$

Finally, we have a contribution from the $u$-channel cut in the initial state described by the diagram in Fig. 8 .

$$
\begin{aligned}
\Delta c_{11}^{\mathrm{qed}}= & \left(\frac{v(-1+2 v)}{v_{1}}-\frac{v^{2} w}{v_{1}}+\frac{2 v_{1} v}{X^{3}}-\frac{2 v}{X^{2}}+\frac{v\left(3-4 v+2 v^{2}\right)}{v_{1} X}\right) \ln \frac{\mu_{F}^{2}}{m^{2}} \\
\Delta c_{11}^{\mathrm{oq}}= & \left(\frac{2 v}{v_{1}}+\frac{4\left(1-2 v+2 v^{2}\right)}{v_{1} v w^{2}}+\frac{4\left(1-4 v+2 v^{2}\right)}{v_{1} w}+\frac{4 v v_{1}}{X^{2}}+\frac{4 v(1-2 v)}{X}\right) \times \\
& \ln \frac{\mu_{F}^{2}}{m^{2}} \\
\Delta c_{11}^{\mathrm{kq}}= & -\Delta c_{11}^{\mathrm{oq}} .
\end{aligned}
$$

The sum of Eqs. (55) and (58), that of Eqs. (56) and (59), and that of Eqs. (57) and (60) are identical to Eqs. (44), (45), and (46) in Ref. [12, respectively.

\subsection{Subtraction terms for $q+\bar{q} \rightarrow Q+\bar{Q}+g$}

The results for the coefficients $\Delta c_{i}$ have the following colour decomposition:

$$
\Delta c_{i}=C_{q}(s) \frac{C_{F}}{2}\left(C_{F} \Delta c_{i}^{\mathrm{cf}}+C_{A} \Delta c_{i}^{\mathrm{ca}}\right)
$$

with

$$
C_{q}(s)=\frac{\alpha_{s}^{3}}{2 N s} .
$$

There are two different cut diagrams contributing to the total result. 


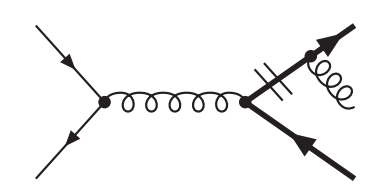

Figure 9: Feynman diagrams representing of $\mathrm{d} \hat{\sigma}^{(0)}(q \bar{q} \rightarrow Q \bar{Q}) \otimes d_{Q \rightarrow Q}^{(1)}(z)$.

$\mathrm{d} \hat{\sigma}^{(0)}(q \bar{q} \rightarrow Q \bar{Q}) \otimes d_{Q \rightarrow Q}^{(1)}(z)$

Figure 9 shows the diagram with a cut in the final state. The $2 \rightarrow 2$ process cross section $\mathrm{d} \hat{\sigma}(q \bar{q} \rightarrow Q \bar{Q})$ is proportional to the function

$$
\tau_{q}(v)=(1-v)^{2}+v^{2}+\frac{2 m^{2}}{s}
$$

which will occur in the results below. Furthermore, for this contribution, the $C_{A}$ parts vanish, i.e., $\Delta c_{i}^{\mathrm{ca}}=0(i=1,2,3,5,10,11)$. Therefore, the final results are proportional to the colour factor $C_{F}^{2}$ :

$$
\begin{aligned}
& \Delta c_{1}=\left[\left(\frac{3}{4}+\ln v\right) \ln \frac{\mu_{F}^{\prime 2}}{m^{2}}+1-\ln v-\ln ^{2} v\right] \times 2 C_{q}(s) \tau_{q}(v) C_{F}^{2} \\
& \Delta c_{2}=\left(\ln \frac{\mu_{F}^{\prime 2}}{m^{2}}-1-2 \ln v\right) \times 2 C_{q}(s) \tau_{q}(v) C_{F}^{2} \\
& \Delta c_{3}=-2 \times 2 C_{q}(s) \tau_{q}(v) C_{F}^{2} \\
& \Delta c_{5}=2 C_{q}(s) C_{F}^{2}\left[v-\frac{2 v v_{1}^{2}}{Y^{3}}+\frac{2 v^{2} v_{1}}{Y^{2}}-\frac{3 v-6 v^{2}+4 v^{3}}{Y}+\frac{m^{2}}{s} 2 v\left(1-\frac{1}{Y}\right)\right] .
\end{aligned}
$$

Finally, we find again

$$
\begin{aligned}
& \Delta c_{10}=\Delta c_{5} \\
& \Delta c_{11}=\frac{1}{2} \Delta c_{5}\left(1-\ln \frac{\mu_{F}^{\prime 2}}{m^{2}}\right) .
\end{aligned}
$$

One can observe the same structure of the results as for $\mathrm{d} \hat{\sigma}^{(0)}(g g \rightarrow Q \bar{Q}) \otimes d_{Q \rightarrow Q}^{(1)}(z)$ given in Sec. 4.1.

Now we turn again to the comparison with the results in Ref. [12. For $\mu_{F}^{\prime}=m$ (and neglecting terms of the order $\mathcal{O}\left(\mathrm{m}^{2} / \mathrm{s}\right)$ ), Eqs. (64)-(69) are identical to Eqs. (51)-(54) in Ref. [12. The parts proportional to $\ln \left(\mu_{F}^{\prime 2} / m^{2}\right)$ in Eqs. (64) and (65) coincide with Eqs. (60) and (61) in Ref. [12. As for $\Delta c_{11}$ in Eq. (69), the part proportional to $\ln \left(\mu_{F}^{\prime 2} / m^{2}\right)$ is in agreement with Eq. (62) in Ref. [12] only after including the contribution from 

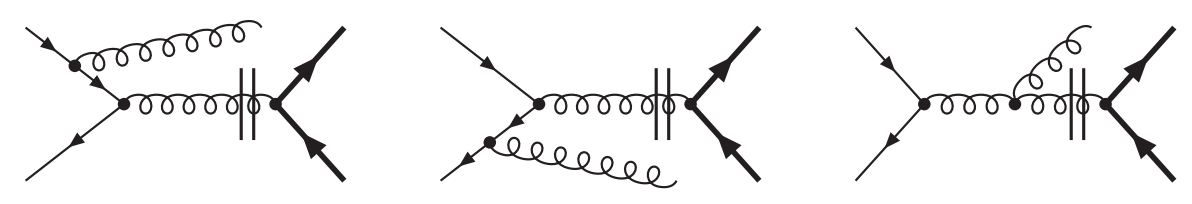

Figure 10: Feynman diagrams representing of $\mathrm{d} \hat{\sigma}^{(0)}(q \bar{q} \rightarrow g g) \otimes d_{g \rightarrow Q}^{(1)}(z)$.

$\mathrm{d} \hat{\sigma}(q \bar{q} \rightarrow g g) \otimes d_{g \rightarrow Q}^{(1)}(z)$, which will be given in the next subsection.

$\mathrm{d} \hat{\sigma}^{(0)}(q \bar{q} \rightarrow g g) \otimes d_{g \rightarrow Q}^{(1)}(z)$

The result for the cut diagram Fig. 10 reads

$$
\begin{aligned}
\Delta c_{11}^{\mathrm{cf}}= & {\left[\frac{2 v\left(3-4 v+2 v^{2}\right)}{v_{1}}+\frac{2\left(1-2 v+2 v^{2}\right)}{w}-\frac{4 v^{3} w}{v_{1}}+\frac{4 v^{3} w^{2}}{v_{1}}-\frac{4 v}{Y}\right] \ln \frac{\mu_{F}^{\prime 2}}{m^{2}}, } \\
\Delta c_{11}^{\mathrm{ca}}= & {\left[4(2-v) v-4 v^{2} w-\frac{4 v v_{1}^{2}}{Y^{3}}+\frac{4\left(3 v-5 v^{2}+2 v^{3}\right)}{Y^{2}}-\frac{2\left(9 v-12 v^{2}+4 v^{3}\right)}{Y}\right] \times } \\
& \ln \frac{\mu_{F}^{\prime 2}}{m^{2}} .
\end{aligned}
$$

Since $\Delta c_{11}$ in Eq. (69) only has a $C_{F}$ part, Eq. (171) is the only contribution to the $C_{A}$ part and hence is in agreement with Eq. (64) in Ref. [12. Furthermore, it is easy to see that the sum of $\Delta c_{11}^{\text {cf }}$ in Eq. (69), taken from the part $\propto \ln \left(\mu_{F}^{\prime 2} / m^{2}\right)$, and $\Delta c_{11}^{\text {cf }}$ in Eq. (70) reproduces Eq. (63) in Ref. [12].

\subsection{Subtraction terms for $g+q \rightarrow Q+\bar{Q}+q$}

The process $g q \rightarrow Q \bar{Q} q$ appears for the first time at NLO. It has the colour decomposition

$$
\Delta c_{i}=C_{g q}(s)\left(C_{F} \Delta c_{i}^{\mathrm{cf}}+C_{A} \Delta c_{i}^{\mathrm{ca}}\right),
$$

with

$$
C_{g q}(s)=\frac{\alpha_{s}^{3}}{2 N s} .
$$

There are two different cut diagrams contributing to the total result. The results for the process $g \bar{q} \rightarrow Q \bar{Q} \bar{q}$ are the same as can be easily seen with the help of the expressions in App. A.3.

$\mathrm{d} \hat{\sigma}^{(0)}(g q \rightarrow g q) \otimes d_{g \rightarrow Q}^{(1)}(z)$

For the cut diagram shown Fig. 11, we find 

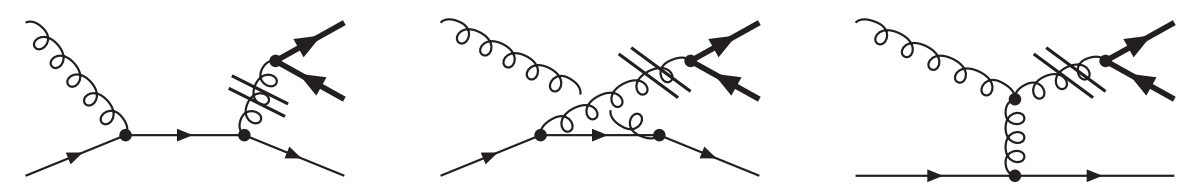

Figure 11: Feynman diagrams representing of $\mathrm{d} \hat{\sigma}^{(0)}(g q \rightarrow g q) \otimes d_{g \rightarrow Q}^{(1)}(z)$.

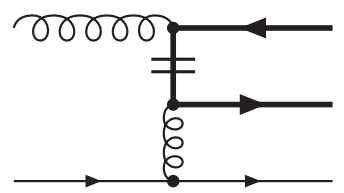

Figure 12: Feynman diagrams representing of $f_{g \rightarrow Q}^{(1)}\left(x_{1}\right) \otimes \mathrm{d} \hat{\sigma}^{(0)}(Q q \rightarrow Q q)$.

$$
\begin{aligned}
& \Delta c_{11}^{\mathrm{cf}}=\left(-2 v^{2}+\frac{1-2 v+2 v^{2}}{2 w}+2 v^{2} w-\frac{v_{1} v}{2 Y^{2}}+\frac{3 v-2 v^{2}}{2 Y}\right) \ln \frac{\mu_{F}^{\prime 2}}{m^{2}}, \\
& \Delta c_{11}^{\mathrm{ca}}=\left(-v^{2}+\frac{v^{2}\left(2-4 v+3 v^{2}\right) w}{v_{1}^{2}}+\frac{2 v^{3}(1-2 v) w^{2}}{v_{1}^{2}}+\frac{2 v^{4} w^{3}}{v_{1}^{2}}+\frac{v}{2 Y}\right) \ln \frac{\mu_{F}^{\prime 2}}{m^{2}} .
\end{aligned}
$$

Equations (74) and (75) are in agreement with Eqs. (69) and (70) in Ref. [12.

$f_{g \rightarrow Q}^{(1)}\left(x_{1}\right) \otimes \mathrm{d} \hat{\sigma}^{(0)}(Q q \rightarrow Q q)$

The contribution of the cut diagram Fig. 12 is given by

$$
\begin{aligned}
& \Delta c_{11}^{\mathrm{cf}}=\frac{1+v^{2}}{2 v_{1}^{2} w}\left(1-2 w+2 w^{2}\right) \ln \frac{\mu_{F}^{2}}{m^{2}}, \\
& \Delta c_{11}^{\mathrm{ca}}=0 .
\end{aligned}
$$

Equation (76) is identical to Eq. (68) in Ref. [12.

\section{Conclusions and discussion}

We have presented a detailed description of the derivation of collinear subtraction terms with the help of mass factorization keeping the heavy-quark mass as a regulator for collinear divergences. As an example, we have considered heavy-quark production in hadron-hadron collisions, which is the most complex case. With one minor exception (see below), we have reproduced all the subtraction terms derived in Ref. [12] and found nice agreement. For a summary of the comparison, see Table 1 Apart from giving additional 


\begin{tabular}{|c|c|c|c|}
\hline channel & & this paper & Ref. 12 \\
\hline$g g \rightarrow Q \bar{Q} g:$ & $\begin{array}{l}\mu_{F}^{\prime}=m \\
\propto \ln \frac{\mu_{F}^{\prime 2}}{m^{2}} \\
\propto \ln \frac{\mu_{F}^{\prime 2}}{m^{2}} \\
\propto \ln \frac{\mu_{F}^{\prime 2}}{m^{2}} \\
\propto \ln \frac{\mu_{F}^{2}}{m^{2}}\end{array}$ & 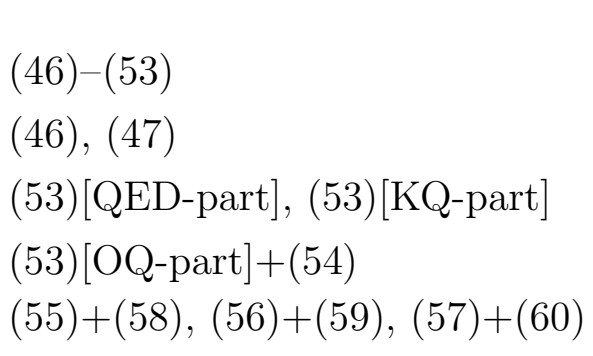 & $\begin{array}{l}(18),(21)-(24) \\
(37),(38) \\
(41),(43) \\
(42) \\
(44),(45),(46)\end{array}$ \\
\hline$q \bar{q} \rightarrow Q \bar{Q} g:$ & $\begin{array}{l}\mu_{F}^{\prime}=m \\
\propto \ln \frac{\mu_{F}^{\prime 2}}{m^{2}} \\
\propto \ln \frac{\mu_{F}^{\prime 2}}{m^{2}} \\
\propto \ln \frac{\mu_{F}^{\prime 2}}{m^{2}}\end{array}$ & $\begin{array}{l}\text { (64) }-(69) \\
\text { (64) },(65) \\
\text { (69) }\left[C_{F}-\text { part }\right]+(70) \\
\text { (711) }\end{array}$ & $\begin{array}{l}(51)-(54) \\
(60),(61) \\
(63) \\
(64)\end{array}$ \\
\hline & $\begin{array}{l}\propto \ln \frac{\mu_{F}^{\prime 2}}{m^{2}} \\
\propto \ln \frac{\mu_{F}^{2}}{m^{2}}\end{array}$ & $\frac{(74)}{(76)},(175)$ & $\begin{array}{l}(69),(70) \\
(68)\end{array}$ \\
\hline
\end{tabular}

Table 1: Collinear subtraction terms for the partonic subprocesses $g+g \rightarrow Q+\bar{Q}+g$, $q+\bar{q} \rightarrow Q+\bar{Q}+g$ and $g+q \rightarrow Q+\bar{Q}+q$ in comparison with the results of Ref. [12. In the second column, ' $\mu_{F}^{\prime}=m$ ' indicates that the final state factorization scale $\mu_{F}^{\prime}$ has to be set to $m$ in the equations in the third column. Furthermore, $\propto \ln \frac{\mu_{F}^{\prime}{ }^{2}}{m^{2}}\left(\propto \ln \frac{\mu_{F}^{2}}{m^{2}}\right)$ refers to those parts of the equations in the third column which are proportional to $\ln \frac{\mu_{F}^{\prime}{ }^{2}}{m^{2}}\left(\ln \frac{\mu_{F}^{2}}{m^{2}}\right)$. The third and forth columns list the equation numbers for the corresponding subtraction terms derived in this paper and in Ref. [12], respectively. Equations combined by a 'plus' sign have to be added.

insight and providing a consistency check of our previous results, this detailed example will be useful for extending the GM-VFN scheme to other processes.

Note however, that some exceptions have been found. (i) In Eq. (25) of Ref. [12, we have found an extra contribution to the coefficient $\Delta c_{1}$ in the $g g \rightarrow Q \bar{Q}$ channel resulting in a modification $\Delta c_{1} \rightarrow \Delta c_{1}-C(s) C_{A} \frac{1}{9} v(1-v)$. This extra piece has its origin in heavyquark loop contributions to the virtual corrections to the Born process $g g \rightarrow Q \bar{Q}$ in Ref. [34] and has no counterpart in the results of Sec. 4.1. However, these terms are absent in the massless limit of the calculation in Refs. 3, 4. Numerically, this modification of $\Delta c_{1}$ turned out to be negligible. (ii) In a publication by two of us [32, subtraction terms for the non-Abelian part of the process $\gamma g \rightarrow Q \bar{Q} g$ have been derived by comparing the zero-mass limit of Ref. [43] with the massless theory of Ref. [44, which do not meet the expectations of mass factorization in Sec. 3. The subtraction terms derived this way 
correctly describe the transition between the two theories. Obviously, if one of the theories uses conventions differing from the conventional $\overline{\mathrm{MS}}$ scheme, the results will not agree with the subtraction terms derived via mass factorization. Whether this is indeed the reason for the discrepancy, can be clarified only with the help of a new full calculation. It is noteworthy that also for the channel $\gamma q \rightarrow Q \bar{Q} q$ a non-vanishing result for the coefficient $\Delta c_{11}$ (see Eq. (78) in Ref. 32]) was found, which would have been zero employing the methods in Sec. 3. In this case, the difference could be traced back to an error in Ref. 44.

\section{Acknowledgment}

We are grateful to I. Bojak for providing his FORTRAN code for the hadroproduction of heavy flavors at NLO, which made this work possible. We would like to thank S. Kretzer and F. Olness for useful discussions. I. S. is grateful to the theory group at Fermilab for the kind hospitality extended to him last summer. During this visit, the present paper was initiated. The work of I. S. was supported by DESY. This work was supported in part by the Bundesministerium für Bildung und Forschung through Grant No. 05 HT4GUA/4.

\section{A Cross sections for $2 \rightarrow 2$ subprocesses}

In this appendix, we list the cross sections for all one-particle-inclusive subprocesses, $a+b \rightarrow c+X$, needed to compute the subtraction terms in Sec. 4 . For brevity, part $X$ of the final state is not written explicitly in the following. We begin with subprocesses occurring in the channel $g+g \rightarrow Q+\bar{Q}+g$, needed in Sec. 4.1.

\section{A.1 Subprocesses in $g+g \rightarrow Q+\bar{Q}+g$}

$$
\begin{aligned}
\frac{\mathrm{d} \hat{\sigma}^{(0)}}{\mathrm{d} \hat{v}}(g g \rightarrow Q) & =\alpha_{s}^{2} \pi \frac{1}{\left(N^{2}-1\right)} \frac{1}{\hat{s}}\left[C_{F}-N \hat{v}(1-\hat{v})\right] \tau(\hat{s}, \hat{v}) \\
\frac{\mathrm{d} \hat{\sigma}^{(0)}}{\mathrm{d} \hat{v}}(g g \rightarrow g) & =\alpha_{s}^{2} \pi \frac{4 N^{2}}{N^{2}-1} \frac{1}{\hat{s}} \frac{(1-x)^{3}}{x^{2}} \\
\frac{\mathrm{d} \hat{\sigma}^{(0)}}{\mathrm{d} \hat{v}}(g Q \rightarrow Q) & =\alpha_{s}^{2} \pi \frac{1}{N^{2}-1} \frac{1}{\hat{s}} \frac{1+(1-\hat{v})^{2}}{\hat{v}} 2 C_{F}\left[C_{F} \hat{v}^{2}+N(1-\hat{v})\right] \frac{1}{\hat{v}(1-\hat{v})} \\
\frac{\mathrm{d} \hat{\sigma}^{(0)}}{\mathrm{d} \hat{v}}(Q g \rightarrow Q) & =\left.\frac{\mathrm{d} \hat{\sigma}^{(0)}}{\mathrm{d} \hat{v}}(g Q \rightarrow Q)\right|_{\hat{v} \leftrightarrow 1-\hat{v}} \\
& =\alpha_{s}^{2} \pi \frac{1}{N^{2}-1} \frac{1}{\hat{s}} \frac{1+\hat{v}^{2}}{1-\hat{v}} 2 C_{F}\left[C_{F}(1-\hat{v})^{2}+N \hat{v}\right] \frac{1}{\hat{v}(1-\hat{v})}
\end{aligned}
$$


where

$$
\begin{aligned}
\tau(\hat{s}, \hat{v}) & =\frac{\hat{v}}{1-\hat{v}}+\frac{1-\hat{v}}{\hat{v}}+\frac{4 m^{2}}{\hat{s} \hat{v}(1-\hat{v})}\left(1-\frac{m^{2}}{\hat{s} \hat{v}(1-\hat{v})}\right), \\
x & =\hat{v}(1-\hat{v}) .
\end{aligned}
$$

\section{A.2 Subprocesses in $q+\bar{q} \rightarrow Q+\bar{Q}+g$}

$$
\begin{aligned}
\frac{\mathrm{d} \hat{\sigma}^{(0)}}{\mathrm{d} \hat{v}}(q \bar{q} \rightarrow Q) & =\alpha_{s}^{2} \pi \frac{C_{F}}{N} \frac{1}{\hat{s}}\left[(1-\hat{v})^{2}+\hat{v}^{2}+\frac{2 m^{2}}{\hat{s}}\right] \\
\frac{\mathrm{d} \hat{\sigma}^{(0)}}{\mathrm{d} \hat{v}}(q \bar{q} \rightarrow g) & =\left.\frac{\mathrm{d} \hat{\sigma}^{(0)}}{\mathrm{d} \hat{v}}(g g \rightarrow Q)\right|_{m \rightarrow 0} \\
& =\alpha_{s}^{2} \pi \frac{1}{N^{2}-1} \frac{1}{\hat{s}}\left[C_{F}-N \hat{v}(1-\hat{v})\right]\left(\frac{\hat{v}}{1-\hat{v}}+\frac{1-\hat{v}}{\hat{v}}\right)
\end{aligned}
$$

A.3 Subprocesses in $g+q \rightarrow Q+\bar{Q}+q$ and $g+\bar{q} \rightarrow Q+\bar{Q}+\bar{q}$

$$
\begin{aligned}
\frac{\mathrm{d} \hat{\sigma}^{(0)}}{\mathrm{d} \hat{v}}(q g \rightarrow g) & =\alpha_{s}^{2} \pi \frac{1}{2 N^{2}} \frac{1}{\hat{s}}\left(2-2 \hat{v}+\hat{v}^{2}\right)\left[\left(N^{2}-1\right) \hat{v}^{2}+2 N^{2}(1-\hat{v})\right] \frac{1}{\hat{v}^{2}(1-\hat{v})}, \\
\frac{\mathrm{d} \hat{\sigma}^{(0)}}{\mathrm{d} \hat{v}}(g q \rightarrow g) & =\left.\frac{\mathrm{d} \hat{\sigma}^{(0)}}{\mathrm{d} \hat{v}}(q g \rightarrow g)\right|_{\hat{v} \leftrightarrow 1-\hat{v}} \\
& =\alpha_{s}^{2} \pi \frac{1}{2 N^{2}} \frac{1}{\hat{s}}\left(1+\hat{v}^{2}\right)\left[\left(N^{2}-1\right) \hat{v}^{2}+2 \hat{v}+\left(N^{2}-1\right)\right] \frac{1}{\hat{v}(1-\hat{v})^{2}}, \\
\frac{\mathrm{d} \hat{\sigma}^{(0)}}{\mathrm{d} \hat{v}}(Q q \rightarrow Q) & =\alpha_{s}^{2} \pi \frac{C_{F}}{N} \frac{1}{\hat{s}} \frac{1+\hat{v}^{2}}{(1-\hat{v})^{2}} \\
\frac{\mathrm{d} \hat{\sigma}^{(0)}}{\mathrm{d} \hat{v}}(Q \bar{q} \rightarrow Q) & =\frac{\mathrm{d} \hat{\sigma}^{(0)}}{\mathrm{d} \hat{v}}(Q q \rightarrow Q) .
\end{aligned}
$$

\section{B Feynman diagrams}

In this appendix we list the bremsstrahlung Feynman diagrams contributing at NLO to the process $p+\bar{p} \rightarrow H+X\left(H\right.$ denotes a heavy meson, $\left.D, D^{\star}, B \ldots\right)$ in the FFNS. They are the basis to generate the cut diagrams Figs. 5 [12 as described in Sec. 3.2. We show separately the subset of Feynman diagrams for $g g \rightarrow Q \bar{Q} g$ which, after replacing one of the incoming gluons by a photon, contribute also to heavy-quark photoproduction, $\gamma+p \rightarrow H+X$. 


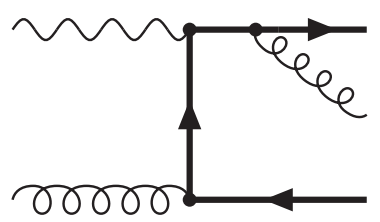

(a)

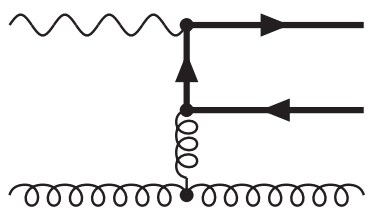

(d)

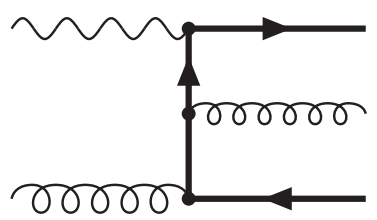

(b)

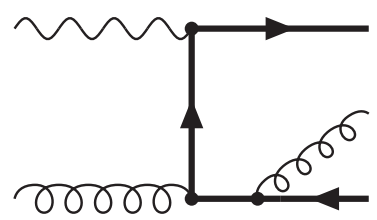

(c)

Figure 13: Feynman diagrams for the NLO gluon bremsstrahlung process $\gamma+g \rightarrow Q+\bar{Q}+$ $g$. Reversing the heavy-quark lines yields the remaining graphs. Diagrams obtained from the ones shown here by replacing the photon with a gluon contribute to $g+g \rightarrow Q+\bar{Q}+g$.

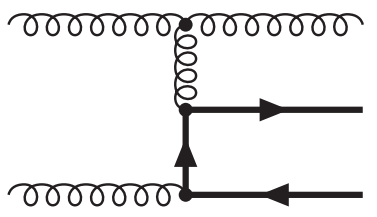

(a)

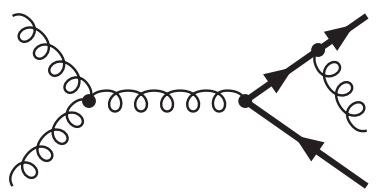

(d)

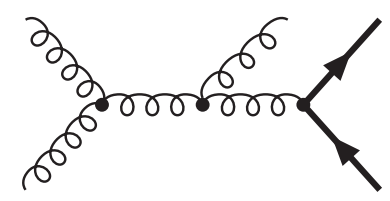

$(\mathrm{g})$

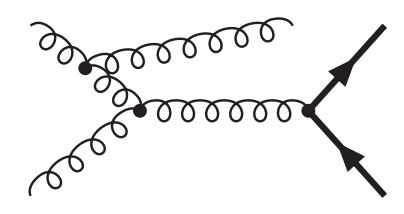

(b)

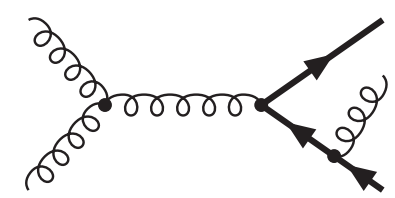

(e)

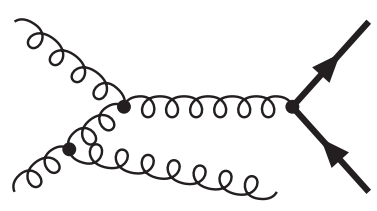

(c)

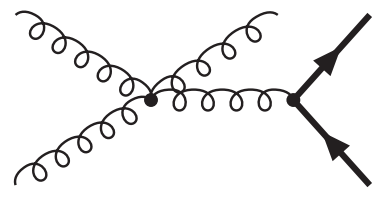

(f)

Figure 14: Additional Feynman diagrams for the NLO gluon bremsstrahlung process $g+g \rightarrow Q+\bar{Q}+g$. Replacing the photons by gluons in Fig. [13 and reversing the heavy-quark lines of part (a) yields the remaining graphs. 


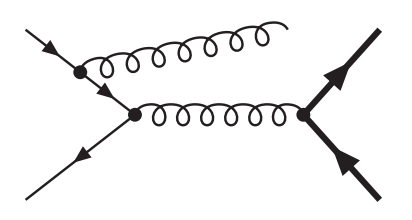

(a)

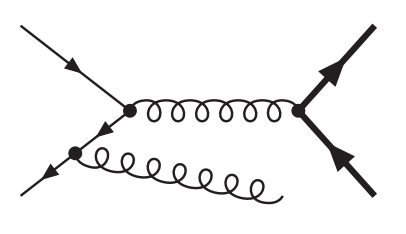

(b)

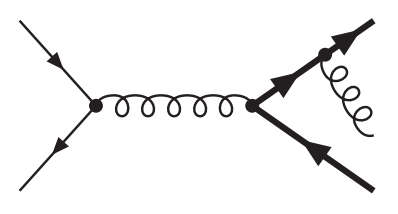

(c)

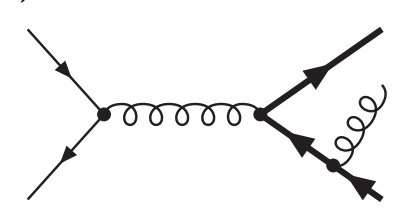

(d)

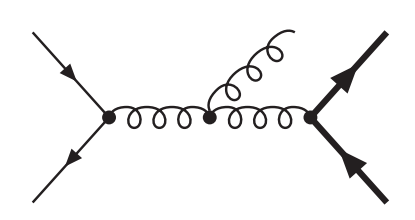

(e)

Figure 15: Feynman diagrams for the NLO gluon bremsstrahlung process $q+\bar{q} \rightarrow Q+\bar{Q}+g$.

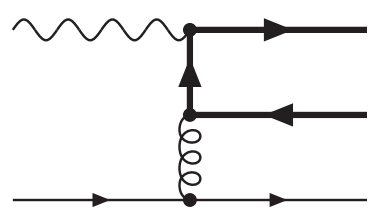

(a)

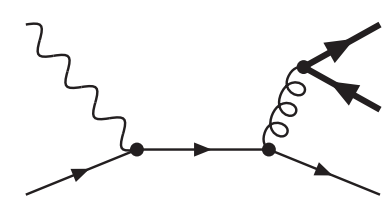

(c)

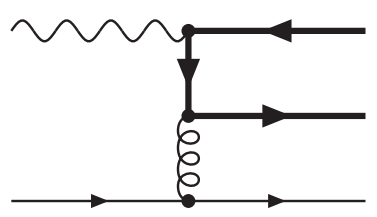

(b)

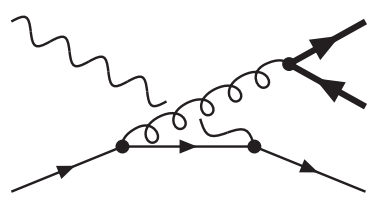

(d)

Figure 16: Feynman diagrams for the NLO light-quark-initiated subprocess $\gamma+q \rightarrow$ $Q+\bar{Q}+q$ : "Bethe-Heitler" graphs (a) and (b), "Compton" graphs (c) and (d). Diagrams obtained from the ones shown here by replacing the photon with a gluon contribute to $g+q \rightarrow Q+\bar{Q}+q$.

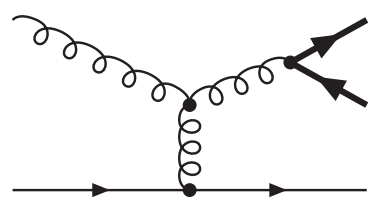

Figure 17: Additional Feynman diagram for the NLO light-quark-initiated subprocess $g+q \rightarrow Q+\bar{Q}+q$. Replacing the photons by gluons in Fig. [16] yields the remaining graphs. 


\section{References}

[1] P. Nason, S. Dawson, and R. K. Ellis, Nucl. Phys. B303 (1988) 607.

[2] P. Nason, S. Dawson, and R. K. Ellis, Nucl. Phys. B327 (1989) 49; Erratum-ibid. B335 (1990) 260.

[3] W. Beenakker, H. Kuijf, W. L. van Neerven, and J. Smith, Phys. Rev. D40 (1989) 54.

[4] W. Beenakker, W. L. van Neerven, R. Meng, G. A. Schuler, and J. Smith, Nucl. Phys. B351 (1991) 507.

[5] J. Amundson, C. Schmidt, W.-K. Tung, and X. Wang, JHEP 10 (2000) 031, hep-ph/0005221.

[6] W.-K. Tung, S. Kretzer, and C. Schmidt, J. Phys. G28 (2002) 983, hep-ph/0110247.

[7] I. Schienbein, Proceedings of the Ringberg Workshop, New Trends in HERA Physics 2003, eds. G. Grindhammer, B. A. Kniehl, G. Kramer and W. Ochs, World Scientific, 2004, p. 197.

[8] B. Mele and P. Nason, Nucl. Phys. B361 (1991) 626.

[9] S. Kretzer and I. Schienbein, Phys. Rev. D58 (1998) 094035, hep-ph/9805233.

[10] S. Kretzer and I. Schienbein, Phys. Rev. D59 (1999) 054004, hep-ph/9808375.

[11] G. Kramer and H. Spiesberger, Eur. Phys. J. C22 (2001) 289, hep-ph/0109167.

[12] B. A. Kniehl, G. Kramer, I. Schienbein, and H. Spiesberger, Phys. Rev. D71 (2005) 014018, hep-ph/0410289.

[13] M. Cacciari and S. Catani, Nucl. Phys. B617 (1002) 253.

[14] J. P. Ma, Nucl. Phys. B506 (1997) 329, hep-ph/9705446.

[15] K. Melnikov and A. Mitov, Phys. Rev. D70 (2004) 034027, hep-ph/0404143.

[16] A. Mitov, hep-ph/0410205.

[17] M. Cacciari and M. Greco, Nucl. Phys. B421 (1994) 530, hep-ph/9311260.

[18] M. Cacciari, M. Greco, B. A. Kniehl, M. Krämer, G. Kramer, and M. Spira, Nucl. Phys. B466 (1996) 173, hep-ph/9512246.

[19] M. Cacciari and M. Greco, Z. Phys. C69 (1996) 459, hep-ph/9505419. 
[20] M. Cacciari and M. Greco, Phys. Rev. D55 (1997) 7134, hep-ph/9702389].

[21] M. Cacciari, M. Greco, and P. Nason, JHEP 05 (1998) 007, hep-ph/9803400.

[22] M. Cacciari, S. Frixione, and P. Nason, JHEP 03 (2001) 006, hep-ph/0102134.

[23] M. Cacciari and P. Nason, Phys. Rev. Lett. 89 (2002) 122003, hep-ph/0204025.

[24] S. Frixione and P. Nason, JHEP 03 (2002) 053, hep-ph/0201281.

[25] M. Cacciari and P. Nason, JHEP 09 (2003) 006, hep-ph/0306212.

[26] M. A. G. Aivazis, F. I. Olness, and W.-K. Tung, Phys. Rev. D50 (1994) 3085, hep-ph/9312318.

[27] M. A. G. Aivazis, J. C. Collins, F. I. Olness, and W.-K. Tung, Phys. Rev. D50 (1994) 3102, hep-ph/9312319.

[28] S. Kretzer and I. Schienbein, Phys. Rev. D56 (1997) 1804, hep-ph/9702296.

[29] F. I. Olness, R. J. Scalise, and W.-K. Tung, Phys. Rev. D59 (1999) 014506, hep-ph/9712494.

[30] J. Binnewies, B. A. Kniehl, and G. Kramer, Z. Phys. C76 (1997) 677, hep-ph/9702408.

[31] G. Kramer, Proceedings of the Ringberg Workshop New Trends in HERA Physics 1999, eds. G. Grindhammer, B. A. Kniehl and G. Kramer, Lecture Notes in Physics 546, Springer, 2000, p. 275.

[32] G. Kramer and H. Spiesberger, Eur. Phys. J. C28 (2003) 495, hep-ph/0302081.

[33] G. Kramer and H. Spiesberger, Eur. Phys. J. C38 (2004) 309, hep-ph/0311062.

[34] I. Bojak and M. Stratmann, Phys. Rev. D67 (2003) 034010, hep-ph/0112276.

[35] I. Bojak, PhD thesis, University of Dortmund, 2000, hep-ph/0005120.

[36] F. Aversa, P. Chiappetta, M. Greco, and J. P. Guillet, Nucl. Phys. B327 (1989) 105.

[37] I. Schienbein, Proceedings of the 12th International Workshop on Deep Inelastic Scattering (DIS 2004), Strbske Pleso, Slovakia, 14-18 Apr 2004, hep-ph/0408036.

[38] M. Krämer, F. I. Olness, and D. E. Soper, Phys. Rev. D62 (2000) 096007, hep-ph/0003035.

[39] J. C. Collins, Phys. Rev. D58 (1998) 094002, hep-ph/9806259.

[40] R. K. Ellis, H. Georgi, M. Machacek, H. D. Politzer, and G. G. Ross, Nucl. Phys. B152 (1979) 285. 
[41] M. Buza, Y. Matiounine, J. Smith, and W. L. van Neerven, Eur. Phys. J. C1 (1998) 301, hep-ph/9612398.

[42] J. C. Collins, F. Wilczek, and A. Zee, Phys. Rev. D18 (1978) 242.

[43] Z. Merebashvili, A. P. Contogouris, and G. Grispos, Phys. Rev. D62 (2000) 114509, Erratum-ibid. D69 (2004) 019901, hep-ph/0007050.

[44] L. E. Gordon, Phys. Rev. D50 (1994) 6753. 